\title{
Floristic composition and phytodiversity status of Sitakunda Ecopark, Chittagong, Bangladesh
}

\author{
Md. Anwarul Islam*, Mohammad Mahfuzur Rahman and Gazi Mosharof Hossain \\ Department of Botany, Jahangirnagar University, Savar, Dhaka-1342, Bangladesh
}

\begin{abstract}
The present study dealt with the exploration and documentation of the floristic composition and phyto-diversity of Sitakunda Eco-park, Chittagong, Bangladesh. A total of 412 vascular plant species under 315 genera belonging to 94 plant families have been recorded from the study area during February, 2013 to April, 2015. Out of these recorded taxa, 330 were dicotyledons, 62 were monocotyledons, 5 were gymnosperms and 15 were pteridophytes. Among those, the maximum 144 species belonged to herbs followed by 138, 75 and 55 species as trees, shrubs and climbers, respectively. The species composition among the plant families varied in plant groups. In dicotyledonous group, Euphorbiaceae appeared to be the largest family with 35 species, whereas Poaceae showed the largest family containing 30 species among monocotyledonous group. The highest values of both Shannon-Weiner and Simpson diversity indices have been observed as 3.82 and 0.98 , respectively to site D during monsoon season, whereas the lowest values 3.19 and 0.95 , respectively of these indices were recorded in site A during summer season.
\end{abstract}

Key words: Floristic composition, phytodiversity, Sitakunda ecopark.

\section{INTRODUCTION}

Bangladesh is located between $20^{\circ} 25^{\prime}$ to $26^{\circ} 38^{\prime}$ North latitude and $88^{\circ} 01^{\prime}$ to $92^{\circ} 42^{\prime}$ East longitude with an area of about 14.4 million hectares (BBS, 1997). The majority of country's land is formed by alluvium soil, which consists mostly of flood plains exceptionally occurring some hilly areas and uplifted land blocks with a sub-tropical monsoon climate (Islam, 2003 and Rashid, 1991). Geographically, Bangladesh is situated near the Indo-Burma region- one of the prime hotspot areas of the world biodiversity (Mittermeier et al., 1998). Due to its unique geo-physical location Bangladesh is exceptionally characterized by a rich biological diversity (Nishat et al., 2002; Hossain, 2001; Barua et al., 2001; Chowdhury, 2001) which harbored approximately 5,000 species of angiosperms (Khan, 1977).

The rich country's biodiversity is increasingly being depleted day by day due to overextraction and destruction of natural ecosystems owing to multifarious anthropogenic activities as well as natural calamities. Lack of proper management practices in some of the natural ecosystems is also one of the remarkable reasons for this degradation. For the

\footnotetext{
* Corresponding author. E-mail: anwarbulu@gmail.com
} 
conservation of natural habitat and its biological diversity, Bangladesh government has declared several categories of protected areas including national parks, ecoparks and botanical gardens, safari parks, wildlife sanctuaries, game reserves, world heritage site etc. following IUCN management criteria.

The Sitakunda ecopark is very important due to its unique habitat and rich biodiversity as well as religious and ecotourism point of view. Naturally growing habitat of a threatened gymnosperm species- the Cycas pectinata is harbored in different locations of Sitakunda ecopark. A number of water falls in this hilly region is the special attraction to the tourists and casual visitors. Few research works on the Sitakunda area were conducted (Alam, 2001; Rahman \& Uddin, 1997; Misbahuzzaman \& Alam, 2006; Shamsuddoha \& Nasir, 2005 etc.). Most of these works covered in the fields of taxonomy, forestry, tourism and management aspects. But the detail ecological research work especially, the floristic composition with their diversity status is still uncompleted. Therefore, the present research on floristic composition and phyto-diversity status of Sitakunda ecopark has been conducted with a view to prepare a comprehensive checklist of vascular plants with their distribution and seasonal fluctuation as well as to assess phyto-diversity status which will help to prepare a plan for sustainable management and eco-friendly conservation of the Sitakunda ecopark.

\section{MATERIALS AND METHODS}

Description of the study site: Present research work was conducted in Sitakuunda Ecpark and adjacent Botanical garden. The ecopark is situated between $22^{\circ} 36^{\prime}$ to $22^{\circ} 39^{\prime}$ North latitude and $91^{\circ} 40^{\prime}$ to $91^{\circ} 42^{\prime}$ East longitude and about 15 to $65 \mathrm{~m}$ above mean sea level. The northern side of the park is Mirsarai upazilla and Pahartali is in the south, Sandwip channel and the Bay of Bengal is in the west and Fatikchari is in the east. Sitakunda ecopark and botanical garden is located along the Dhaka-Chittagong highway in Sitakunda upazilla of Chittagong district. Four main sites were selected covering the whole ecopark area on the bases of gradual hill altitude and geo-spatial location, which were- site-A (Ecopark's office region with an average 220 feet high from the mean sea level), site-B (Suptodhara region with an average 600 feet high from the mean sea level), site-C (Sohosrodhara region with an average 1100 feet high from the mean sea level) and site-D (Chandranath temple region with an average 1400 feet high from the mean sea level). Each site was again divided into three sample area as sub-sites, namely sub-site 1 (upper-slope at the hill top), sub-site 2 (mid-slope of the hill) and sub-site 3 (lower-slope at the hill foot) according to the gradual hill slope. So, a total of 12 sub-sites were selected for the present research investigation.

Field survey, sample collection and identification: A total of nine field trips with seven days duration of each were conducted during three prominent sampling seasons of the year (viz., Rainy, Summer and Winter) from 2013 to 2015. Vascular plants were collected following standard quadrat method (Braun-Blanquet, 1932; Raunkiaer, 1934). The quadrat size, like $2 \mathrm{~m} \times 2 \mathrm{~m}$ for herbs and grasses, $5 \mathrm{~m} \times 5 \mathrm{~m}$ for shrubs and $10 \mathrm{~m} \times 10 \mathrm{~m}$ for trees were standardized on the basis of species-area-curve method (Cain, 1938). In each 
sub-site, ten plots with $10 \mathrm{~m} \times 10 \mathrm{~m}$ sized-quadrat were selected and other smaller sizedquadrats were placed within the big one for collecting the respective plants group. Collected plant specimens were properly processed using standard herbarium techniques (Hyland, 1972; Jain and Raw, 1977 and Alexiades, 1996) for voucher specimens, which have been preserved in Plant Ecology and Environment Laboratory, Jahangirnagar University. All plant specimens collected from the study area were identified through consulting the experts and matching with relevant voucher specimens preserved at Jahangirnagar University Herbarium (JUH) and Bangladesh National Herbarium (DACB), taxonomic descriptions and keys available in the relevant literatures (Prain, 1903b; Hooker, 1973; Wu et al., 2003; Siddiqui, 2007a, b; Ahmed, 2008a, b, c, d; Ahmed, 2009a, b; and type images available in the websites of different international herbaria). Updated nomenclatural information was incorporated through data bases including IPNI (2008) and TROPICOS (2010). Scientific name with original citation, family name, local name, major plant group, habit, and one representative specimen number have been provided. The genera under each family and the species under each genus are arranged alphabetically.

Vegetation analysis: Simpson's and Shannon-Weiner diversity indices were calculated by the following formulae-

Simpson's diversity index (Simpson, 1949) was calculated by the following formulae described by Kent and Coker (1992)-

$$
\begin{aligned}
& \text { Simpson's Diversity Index: } \quad 1-\mathrm{D}=1-\frac{\sum \mathrm{n}(\mathrm{n}-1)}{\mathrm{N}(\mathrm{N}-1)} \\
& \text { where, } \mathrm{n} \text { is the total no. of individuals of each species, } \\
& \mathrm{N} \text { is the total no. of organisms of all species. }
\end{aligned}
$$

The Shannon-Wiener diversity index (Shannon and Wiener, 1963) was calculated by the following formulae described by Kent and Coker (1992)-

$$
\begin{aligned}
& \text { Shannon-Weiner Diversity Index: } \mathrm{H}^{\prime}=-\Sigma \mathrm{pi} \ln \mathrm{pi} \\
& \text { where, pi is the proportion of individuals or the } \\
& \text { abundance of the ith species expressed as a proportion of } \\
& \text { total cover, } \\
& \text { ln is Log base } \mathrm{n}_{\mathrm{n}} .
\end{aligned}
$$

Statistical Analysis: Data were statistically analyzed using SPSS software (version 16.0). One way ANOVA (DMRT) was used to test for significant differences $(\mathrm{P}<0.05)$ for marginal means of variables.

\section{RESULTS AND DISCUSSION}

A total of 412 vascular plant species under 315 genera belonging to 94 plant families have been documented from the study area (Table 1). Among the enumerated species, 392 are angiosperms, 5 are gymnosperms and rests of 15 are pteridophytes. Out of the recorded 
angiospermic flora, the dicotyledons (magnoliopsida) group is represented by 330 species belonging to 252 genera under 67 plant families, whereas the monocotyledons (liliopsida) group occupied 62 species under 49 genera and 14 families. In this study, the gymnosperms consisted of 5 species belonging to 4 genera under 4 families whereas the pteridopytic flora revealed the occurrence of 15 species under 10 genera and 9 families. Among the total species, 144, 138, 75 and 55 species respectively represented as herbs, trees, shrubs and climbers. The number of species in plant families varried from 1 to 35, where 20 families are monospecific representing single species. The most 10 dominant families containing maximum number of plant species of the study area are Euphorbiaceae, Poaceae, Rubiaceae, Fabaceae, Caesalpiaceae, Verbenaceae, Asteraceae, Mimosaceae, Araceae and Arecaceae (Fig. 1). In dicotyledonous group, Euphorbiaceae appeared to be the largest family containing 35 species under 23 genera followed by Rubiaceae (27 sp), Fabaceae (20 sp), Caesalpiniaceae (14 sp), Asteraceae (13 sp), Verbinaceae (09 sp), Scrophulariaceae (09 sp), Moraceae (09 sp), Mimosaceae (08 sp) and Combretaceae (05 sp). In monocotyledonous group, Poaceae is the largest family having with 30 species under 23 genera followed by Araceae (08 sp), Arecaceae (06 sp), Cyperaceae (06 sp), Zingeberaceae (03 sp) and Liliaceae (02 sp). Seven largest genera of dicotyledons are Ficus (6 sp.), Lindernia (6 sp.), Senna (5 sp.), Syzygium (5 sp.), Crotalaria (4 sp.), Terminalia (4 sp.) and Zyzyphus (4 sp.); while in monocotyledons important genera are- Calamuus (4 species), Cyperus (4 sp.), Setaria (3 sp.), Themenda (3 sp.), and Sporobolus (2 sp.). The highest number of species (324) was recorded during monsoon season followed by 256 and 183 species during winter and summer seasons respectively (Fig. 3). Uddin and Rahman (1997) surveyed the angiospermic flora of Sitakunda in Chittagong, Bangladesh. They reported 203 dicotyledones in the area. But no such work has been carried out so far for Sitakunda Eco-park. Uddin and Hassan (2010) recorded 374 species at Lawachara National Park, Arefin et al. (2011) recorded 186 species at Satchhari National Forest. Hadi and Rahman (2013) recorded 203 species at Tilagarh Ecopark, Sylhet.

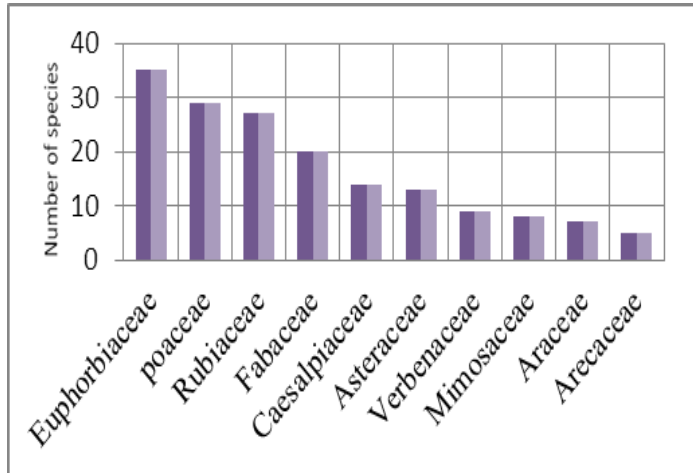

Fig. 1. Ten dominant plant families containing maximum species

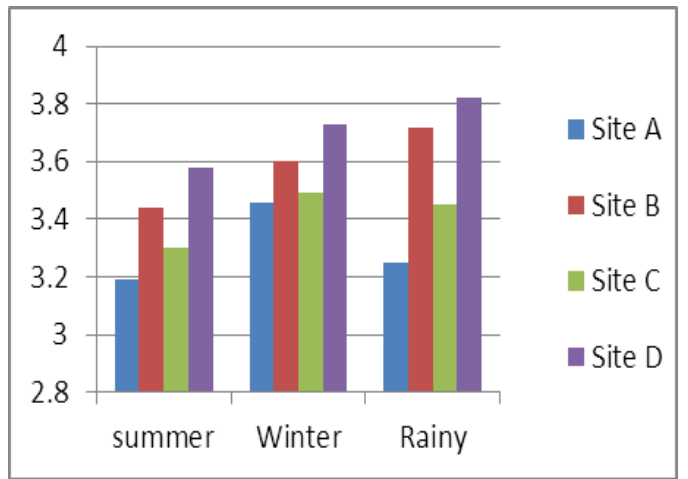

Fig. 2(A). Season-wise Shannon-Weiner diversity index 


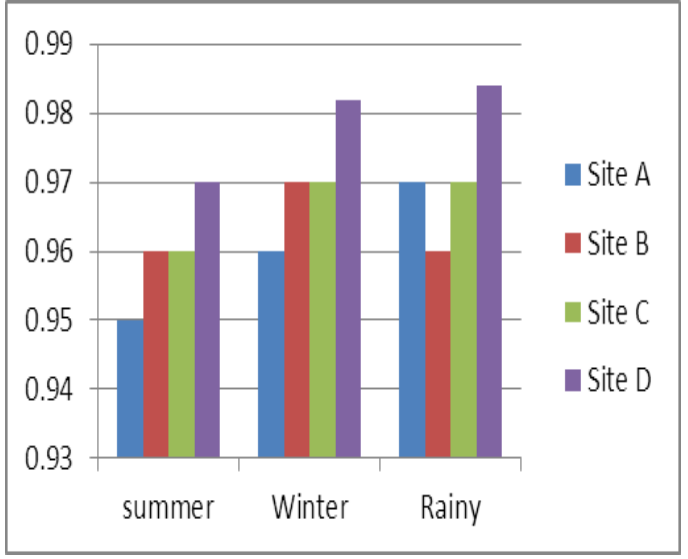

Fig. 2(B). Season-wise Simpson diversity index

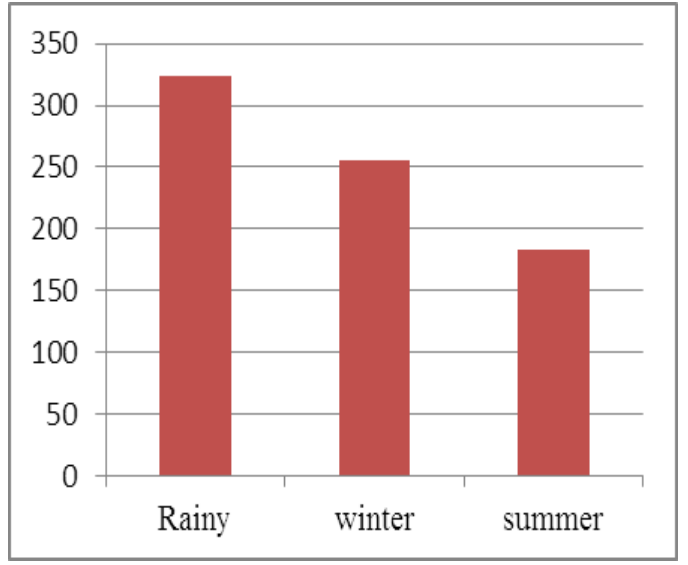

Fig. 3. Seasonal variation of plant species number

The Simpson's and Shannon-Weiner diversity indices in the present study varied from 0.95 to 0.98 and 3.19 to 3.82 , respectively. The highest values of Simpson's and Shannon-Weiner diversity indices 0.98 and 3.82 respectively were observed in site-D during monsoon season (Fig. 2a and 2b), whereas the lowest values 0.95 and 3.19 were in site A during summer season. In site B, the highest values of Simpson's and ShannonWeiner diversity indices (0.96 and 3.72) observed during monsoon season, followed by 0.97 and 3.60, 0.96 and 3.44 during winter and summer seasons respectively. Similarly in site C, the highest value of Simpson's and Shannon-Weiner diversity indices were 0.97 and 3.49 respectively recorded during winter season which was 0.97 and 3.45 during monsoon season and the lowest values were 0.96 and 3.30 respectively during summer season (Fig. 2a and Fig. 2b).

The present findings indicated that the average values of both Simpson's and ShannonWeiner diversity indices within the selected sites and sub-sites did not show significant difference, whereas these variation among the seasons showed significant difference at $5 \%(\alpha=0.05)$ level after DMRT (Table 2). Here, the average values of both the indices during summer season showed the lowest and found significant difference from the average values that observed during winter and rainy reasons. But these values in winter and rainy seasons did not show significant variation at $5 \%$ level by DMRT.

Sitakunda is the first eco-park of Bangladesh. It is highly rich in plant diversity. Floristic composition of Sitakunda ecopark was studied to know the present phytodiversity status in this important natural forest ecosystem. Significant difference of diversity of plants was observed in different seasons. Highest number of species was recorded during rainy season because of growing various types of undergrowth in that season, many of which were not found to grow during winter and summer season. The highest diversity index (DI) observed in site D during Rainy season. The lowest diversity index was observed in site A during summer season. Research findings obtained through this piece of research 
will be helpful for the sustainable management and conservation of phytodiversity resources of Sitakunda ecopakr of Bangladesh.

Table 2. DMRT analysis of diversity indices of selected sites, sub-sites and seasons

\begin{tabular}{ll|c|c}
\hline \multirow{2}{*}{ Sites/Subsites/Seasons } & \multicolumn{2}{c}{ Diversity index value } \\
\cline { 3 - 4 } \multirow{3}{*}{ Sites } & Site-A & Shannon-Weiner & Simpson's \\
& Site-B & $3.36 \mathrm{a}$ & $0.961 \mathrm{a}$ \\
& Site-C & $3.39 \mathrm{a}$ & $0.958 \mathrm{a}$ \\
\multirow{5}{*}{ Subsites } & Site-D & $3.29 \mathrm{a}$ & $0.958 \mathrm{a}$ \\
& Sub-site-1 & $3.49 \mathrm{a}$ & $0.967 \mathrm{a}$ \\
& Sub-site-2 & $3.42 \mathrm{a}$ & $0.96 \mathrm{a}$ \\
\multirow{3}{*}{ Seasons } & Sub-site-3 & $3.36 \mathrm{a}$ & $0.96 \mathrm{a}$ \\
& Summer & $3.36 \mathrm{a}$ & $0.96 \mathrm{a}$ \\
& Winter & $3.10 \mathrm{a}$ & $0.949 \mathrm{a}$ \\
& Rainy & $3.49 \mathrm{~b}$ & $0.965 \mathrm{~b}$ \\
& & $3.55 \mathrm{~b}$ & $0.968 \mathrm{~b}$ \\
\hline
\end{tabular}

Note: Values in the same column that do not share common letters are significantly different at $5 \%(\alpha=0.05)$ level among the sites and seasons after DMRT.

Table 1. Comprehensive checklist of plant species recorded from Sitakunda ecopark of Bangladesh

\begin{tabular}{|c|c|c|c|c|c|c|}
\hline $\begin{array}{c}\text { S.N } \\
\text {. }\end{array}$ & Scientific name & Local name & Family & Type & Habit & Acc. No. \\
\hline 1 & Abroma augusta (L.) L. f. & Ulot kombol & Sterculiaceae & $\mathrm{D}$ & $\mathrm{S}$ & Anwar-0869 \\
\hline 2 & Abutilon indicum (L.) Sweet & Petari & Malvaceae & $\mathrm{D}$ & $\mathrm{H}$ & Anwar-0949 \\
\hline 3 & Abutilon persicum (Burm. f.) Merr. & $\begin{array}{l}\text { Peach } \\
\text { abutilon }\end{array}$ & Malvaceae & $\mathrm{D}$ & $\mathrm{H}$ & Anwar-0354 \\
\hline 4 & $\begin{array}{l}\text { Acacia auriculiformis A. Cunn. ex Benth. } \\
\& \text { Hook. }\end{array}$ & Akashmoni & Mimosaceae & $\mathrm{D}$ & $\mathrm{T}$ & Anwar-0870 \\
\hline 5 & $\begin{array}{l}\text { Acacia nilotica (L.) Delile subsp.indica } \\
\text { (Benth.) Brenan }\end{array}$ & Babla & Mimosaceae & $\mathrm{D}$ & $\mathrm{T}$ & Anwar-0891 \\
\hline 6 & Acalypha indica $\mathrm{L}$. & muktajhuri & Euphorbiaceae & $\mathrm{D}$ & $\mathrm{H}$ & Anwar-0901 \\
\hline 7 & Achyranthes aspera $\mathrm{L}$. & Apang & Amaranthaceae & $\mathrm{D}$ & $\mathrm{H}$ & Anwar-0872 \\
\hline 8 & Actephila excelsa (Dalz.) Muell.-Arg & Achamasi & Euphorbiaceae & $\mathrm{D}$ & S & Anwar-0071 \\
\hline 9 & Adenia trilobata (Roxb.) Engl. & Akandaphal & Passifloraceae & $\mathrm{D}$ & $\mathrm{CH}$ & Anwar-0311 \\
\hline 10 & Adiantum capillus-veneris L. & Bidda pata & Adiantaceae & $\mathrm{F}$ & FH & Anwar-0973 \\
\hline 11 & Adiantum philippense $\mathrm{L}$. & Bidda pata & Adiantaceae & $\mathrm{F}$ & $\mathrm{H}$ & Anwar-1105 \\
\hline 12 & Aegle marmelos (L.) Corr. & Bel & Rutaceae & $\mathrm{D}$ & $\mathrm{T}$ & Anwar-0895 \\
\hline 13 & Aeschynomene indica $\mathrm{L}$. & Shola & Fabaceae & D & $\mathrm{H}$ & Anwar-0988 \\
\hline 14 & Ageratum conyzoides $\mathrm{L}$. & Fulkuri & Asteraceae & $\mathrm{D}$ & $\mathrm{H}$ & Anwar-1081 \\
\hline 15 & Aglaonema hookerianum Schott & Aglaonema & Araceae & M & $\mathrm{H}$ & Anwar-0618 \\
\hline 16 & Albizia chinensis (Osb.) Merr & Chkua-koroi & Mimosaceae & $\mathrm{D}$ & $\mathrm{T}$ & Anwar-0902 \\
\hline 17 & Albizia lebbeck (L.) Benth. \& Hook & Kalo-koroi & Mimosaceae & $\mathrm{D}$ & $\mathrm{T}$ & Anwar-0876 \\
\hline 18 & Albizia procera (Roxb.) Benth. & Sada-koroi & Mimosaceae & $\mathrm{D}$ & $\mathrm{T}$ & Anwar-0899 \\
\hline 19 & $\begin{array}{l}\text { Aleuritopteris albomarginata (C. B. } \\
\text { Clarke) Ching }\end{array}$ & Silver fern & Sinopteridaceae & $\mathrm{F}$ & $\mathrm{FH}$ & Anwar-1058 \\
\hline 20 & Aleuritopteris grisea (Blanford) Panigr & Silver fern & Sinopteridaceae & $\mathrm{F}$ & FH & Anwar-1027 \\
\hline 21 & Alpinia nigra (Gaertn.) Burtt. & Kulanjan & Zingiberaceae & M & $\mathrm{H}$ & Anwar-1028 \\
\hline 22 & $\begin{array}{l}\text { Alternanthera philoxeroides (Mart.) } \\
\text { Griseb }\end{array}$ & Helencha & Amaranthaceae & $\mathrm{D}$ & $\mathrm{H}$ & Anwar-0897 \\
\hline
\end{tabular}




\begin{tabular}{|c|c|c|c|c|c|c|}
\hline 23 & $\begin{array}{l}\text { Alternanthera sessilis (L.) R. Br. ex Roem. } \\
\& \text { Schult }\end{array}$ & Sanchi & Amaranthaceae & $\mathrm{D}$ & $\mathrm{H}$ & Anwar-0911 \\
\hline 24 & Amaranthus spinosus $\mathrm{L}$. & Kata-notey & Amaranthaceae & $\mathrm{D}$ & $\mathrm{H}$ & Anwar-0919 \\
\hline 25 & Amaranthus viridis $\mathrm{L}$. & Notey & Amaranthaceae & $\mathrm{D}$ & $\mathrm{H}$ & Anwar-0294 \\
\hline 26 & Amorphophallus bulbifer (Roxb.) Blume & Jungle Ool & Araceae & M & $\mathrm{H}$ & Anwar-1101 \\
\hline 27 & $\begin{array}{l}\text { Amorphophallus longituberosus (Engl.) } \\
\text { Engl. et Gehrm }\end{array}$ & Ool & Araceae & M & $\mathrm{H}$ & Anwar-0015 \\
\hline 28 & Ampelocissus latifolia (Roxb.) Planch & Gowalia lata & Vitaceae & $\mathrm{D}$ & $\mathrm{CH}$ & Anwar-1130 \\
\hline 29 & Ampelygonum salarkhanii Hassan & $\begin{array}{l}\text { Girishobhon } \\
\text { sak }\end{array}$ & Polygonaceae & $\mathrm{D}$ & $\mathrm{H}$ & Anwar-0686 \\
\hline 30 & Andrographis paniculata (Burm. f.) Wall & Kalomegh & Acanthaceae & $\mathrm{D}$ & $\mathrm{H}$ & Anwar-0599 \\
\hline 31 & Angiopteris evecta (G.Forst.) Hoffm. & Dhekia shak & Angiopteridaceae & $\mathrm{F}$ & $\mathrm{H}$ & Anwar-0093 \\
\hline 32 & Anisomeles indica (L.) O. Kuntze & Gobura & Lamiaceae & $\mathrm{D}$ & $\mathrm{H}$ & Anwar-0540 \\
\hline 33 & Annona reticulata $\mathrm{L}$. & Sarifa & Annonaceae & $\mathrm{D}$ & ST & Anwar-0984 \\
\hline 34 & Annona squamosa $\mathrm{L}$. & Ata-sharifa & Annonaceae & $\mathrm{D}$ & ST & Anwar-0082 \\
\hline 35 & Antidesma acidum Retz. & Multa & Euphorbiaceae & $\mathrm{D}$ & $\mathrm{T}$ & Anwar-0666 \\
\hline 36 & Antidesma ghaesembilla Gaertn & Shialboka & Euphorbiaceae & $\mathrm{D}$ & $\mathrm{T}$ & Anwar-0937 \\
\hline 37 & Antidesma roxburghii Wall, ex Tulasne & $\begin{array}{l}\text { Boro } \\
\text { Shialboka }\end{array}$ & Euphorbiaceae & $\mathrm{D}$ & $\mathrm{T}$ & Anwar-1125 \\
\hline 38 & Aphania danura (Roxb.) Radlk. & Goda-horina & Sapindaceae & $\mathrm{D}$ & ST & Anwar-1077 \\
\hline 39 & Aporosa dioica (Roxb.) Muell.-Arg. & Patkharolla & Euphorbiaceae & $\mathrm{D}$ & $\mathrm{T}$ & Anwar-1131 \\
\hline 40 & Araucaria cookie Hook. & Cooki pine & Araucariaceae & $\mathrm{G}$ & ST & Anwar-1113 \\
\hline 41 & Araucaria excels $\mathrm{R}$. Br. & $\begin{array}{l}\text { Cristmas } \\
\text { tree }\end{array}$ & Araucariaceae & $\mathrm{G}$ & ST & Anwar-0921 \\
\hline 42 & Ardisia solanacea (Poir.) Roxb. & Chaoldhoa & Myrsinaceae & $\mathrm{D}$ & S & Anwar-0989 \\
\hline 43 & $\begin{array}{l}\text { Argyreia capitiformis (Poir.) van Cheek } \\
\text { Oostr }\end{array}$ & Doitta lati & Convolvulaceae & $\mathrm{D}$ & $\mathrm{CH}$ & Anwar-0355 \\
\hline 44 & Aristolochia tagala Cham. & Ishwarmul $t$ & Aristolochiaceae & $\mathrm{D}$ & $\mathrm{CH}$ & Anwar-0864 \\
\hline 45 & Artabotrys hexapetalus (L.f.) Bhandari & Kathalichapa & a Annonaceae & $\mathrm{D}$ & S & Anwar-1111 \\
\hline 46 & Artocarpus chama Buch. Ham. ex Wall. & Chhapalish & Moraceae & $\mathrm{D}$ & $\mathrm{T}$ & Anwar-1095 \\
\hline 47 & Artocarpus heterophyllus Lamk. & Kathal & Moraceae & $\mathrm{D}$ & $\mathrm{T}$ & Anwar-0913 \\
\hline 48 & Asclepias curassavica $\mathrm{L}$. & $\begin{array}{l}\text { Blood } \\
\text { flower }\end{array}$ & Asclepiadaceae & $\mathrm{D}$ & $S$ & Anwar-0097 \\
\hline 49 & Atylosia scarabaeoides (L.) Baker & Banor kolai & Fabaceae & $\mathrm{D}$ & $\mathrm{CH}$ & Anwar-1121 \\
\hline 50 & Averrhoa bilimbi L. & Bilimbi & Oxalidaceae & $\mathrm{D}$ & ST & Anwar-0942 \\
\hline 51 & Axonopus compressus (Sw.) P. Beauv. & Chapra ghas & Poaceae & M & $\mathrm{H}$ & Anwar-1129 \\
\hline 52 & Azadirachta indica A. Juss & Neem & Meliaceae & $\mathrm{D}$ & $\mathrm{T}$ & Anwar-0903 \\
\hline 53 & Baccaurea ramiflora Lour. & Latkon & Euphorbiaceae & $\mathrm{D}$ & ST & Anwar-0875 \\
\hline 54 & Bambusa balcooa Roxb. & Borak Bans & Poaceae & M & $\mathrm{T}$ & Anwar-0981 \\
\hline 55 & Barringtonia acutangula (L.) Gaertn. & Hijol & Lecythidaceae & $\mathrm{D}$ & $\mathrm{T}$ & Anwar-1104 \\
\hline 56 & Bauhinia purpurea $\mathrm{L}$. & $\begin{array}{l}\text { Rakto } \\
\text { kanchon }\end{array}$ & Caesalpiniaceae & $\mathrm{D}$ & $\mathrm{T}$ & Anwar-1084 \\
\hline 57 & Bauhinia scandens L. & Swarpa gach & Caesalpiniaceae & $\mathrm{D}$ & $\mathrm{CS}$ & Anwar-0665 \\
\hline 58 & Biophytum reinwardtii (Zucc.) Klotzsch & $\begin{array}{l}\text { Banna } \\
\text { ringati }\end{array}$ & Oxalidaceae & $\mathrm{D}$ & $\mathrm{H}$ & Anwar-0435 \\
\hline 59 & Bischofia javanica Blume & Kainjal & Euphorbiaceae & $\mathrm{D}$ & $\mathrm{T}$ & Anwar-0978 \\
\hline 60 & Blumea lacera (Burm. f.) DC. & Kukursunga & Asteraceae & $\mathrm{D}$ & $\mathrm{H}$ & Anwar-1056 \\
\hline 61 & Blumea membranacea Wall. ex DC. & Kukhshi & Asteraceae & $\mathrm{D}$ & $\mathrm{H}$ & Anwar-1110 \\
\hline 62 & Blumea procera DC. & Not known & Asteraceae & $\mathrm{D}$ & $\mathrm{H}$ & Anwar-0426 \\
\hline 63 & Boehmeria macrophylla Hornem. & Ulichara & Urticaceae & $\mathrm{D}$ & $\mathrm{H}$ & Anwar-1117 \\
\hline 64 & Bombax ceiba L. & Shimul & Bombacaceae & $\mathrm{D}$ & $\mathrm{T}$ & Anwar-1067 \\
\hline 65 & Bougainvillea glabra Choisy & Baganbilash & Nyctaginaceae & $\mathrm{D}$ & $\mathrm{CS}$ & Anwar-0936 \\
\hline 66 & Brachiaria decumbens Stapf & $\begin{array}{l}\text { Songket } \\
\text { ghas }\end{array}$ & Poaceae & M & $\mathrm{H}$ & Anwar-0414 \\
\hline 67 & Breynia retusa (Dennst.) Alston & Silpati & Euphorbiaceae & $\mathrm{D}$ & S & Anwar-0012 \\
\hline
\end{tabular}




\begin{tabular}{|c|c|c|c|c|c|c|}
\hline 68 & $\begin{array}{l}\text { Breynia vitis-idaea (Burm. f.) C.E.C. } \\
\text { Fischer }\end{array}$ & Vita salpoti & Euphorbiaceae & $\mathrm{D}$ & $\mathrm{S}$ & Anwar-0286 \\
\hline 69 & Bridelia retusa (L.) A. Juss. & Kata kushui & Euphorbiaceae & $\mathrm{D}$ & ST & Anwar-1115 \\
\hline 70 & Bridelia stipularis (L.) Blume & Pat-khowi & Euphorbiaceae & $\mathrm{D}$ & SS & Anwar-0508 \\
\hline 71 & Bryophyllum pinnatum (Lamk.) Oken & Pathor kuchi & Crasulaceae & $\mathrm{D}$ & $\mathrm{H}$ & Anwar-0974 \\
\hline 72 & Bursera serrata Wall. ex Colebr. & Bhadi & Burserraceae & $\mathrm{D}$ & $\mathrm{T}$ & Anwar-0489 \\
\hline 73 & Butea monosperma (Lamk.) Taub. & Polash & Fabaceae & $\mathrm{D}$ & $\mathrm{T}$ & Anwar-1100 \\
\hline 74 & Byttneria pilosa Roxb. & $\begin{array}{l}\text { Harvanga } \\
\text { lata }\end{array}$ & Sterculiaceae & $\mathrm{D}$ & SS & Anwar-0758 \\
\hline 75 & Caesalpinia crista $\mathrm{L}$. & Nata & Caesalpiniaceae & $\mathrm{D}$ & $\mathrm{CS}$ & Anwar-0664 \\
\hline 76 & Caesalpinia pulcherrima (L.) Swartz & Radhachura & Caesalpiniaceae & $\mathrm{D}$ & $\mathrm{S}$ & Anwar-1061 \\
\hline 77 & Calamus erectus Roxb. & Kadam bet & Arecaceae & M & CS & Anwar-1029 \\
\hline 78 & Calamus gracilis Roxb. & Kiring bet & Arecaceae & M & CS & Anwar-1122 \\
\hline 79 & Calamus guruba Buch.-Ham. ex Martius & Jali bet & Arecaceae & M & $\mathrm{CS}$ & Anwar-0966 \\
\hline 80 & Calamus viminalis Willd. & Bara bet & Arecaceae & M & $\mathrm{CS}$ & Anwar-1033 \\
\hline 81 & Callicarpa arborea Roxb. & Barmala & Verbenaceae & $\mathrm{D}$ & $\mathrm{T}$ & Anwar-0713 \\
\hline 82 & Callistemon citrinus (Curtis) Skeels & Bottle brush & Myrtaceae & $\mathrm{D}$ & $\mathrm{T}$ & Anwar-0958 \\
\hline 83 & Calophyllum inophyllum L. & Pipul/ punial & Clusiaceae & $\mathrm{D}$ & $\mathrm{T}$ & Anwar-1063 \\
\hline 84 & Calotropis procera (Ait.) R. Br. & Akond & Asclepiadaceae & $\mathrm{D}$ & $\mathrm{S}$ & Anwar-1114 \\
\hline 85 & $\begin{array}{l}\text { Cananga odorata (Lamk.) Hook. f. \& } \\
\text { Thorn }\end{array}$ & $\begin{array}{l}\text { Sugondhi } \\
\text { gach }\end{array}$ & Annonaceae & $\mathrm{D}$ & $\mathrm{CH}$ & Anwar-1091 \\
\hline 86 & Canscora decussata (Roxb.) Schult & Dhankuni & Gentianaceae & $\mathrm{D}$ & $\mathrm{H}$ & Anwar-0542 \\
\hline 87 & Catharanthus roseus (L.) G.Don & Nayan tara & Apocynaceae & $\mathrm{D}$ & $\mathrm{H}$ & Anwar-1088 \\
\hline 88 & Canthium angustifolium Roxb. & Kantanali & Rubiaceae & $\mathrm{D}$ & S & Anwar-0826 \\
\hline 89 & Cardiospermum halicacabum $\mathrm{L}$. & Sibjhul & Sapindaceae & $\mathrm{D}$ & S & Anwar-1045 \\
\hline 90 & Caryota mitis Lour. & Ban supari & Araceae & M & S & Anwar-1070 \\
\hline 91 & Cassia sophera $\mathrm{L}$. & $\begin{array}{l}\text { Chotokolkas } \\
\text { undh }\end{array}$ & Caesalpiniaceae & $\mathrm{D}$ & $\mathrm{S}$ & Anwar-1102 \\
\hline 92 & Casuarina equisetifolia Forst. & Jhau & Casuarinaceae & $\mathrm{D}$ & $\mathrm{T}$ & Anwar-0898 \\
\hline 93 & Cayratia japonica (Thunb.) Gagnep. & $\begin{array}{l}\text { Japani } \\
\text { goalilata }\end{array}$ & Vitaceae & $\mathrm{D}$ & $\mathrm{CH}$ & Anwar-1074 \\
\hline 94 & Celtis timorensis Span. & Timur lata & Ulmacae & $\mathrm{D}$ & ST & Anwar-0654 \\
\hline 95 & Centella asiatica (L.) Urban & Thankuni & Apiaceae & $\mathrm{D}$ & $\mathrm{H}$ & Anwar-0877 \\
\hline 96 & Centotheca lappacea (L.) Desv. & Centu ghas & Poaceae & M & $\mathrm{H}$ & Anwar-1009 \\
\hline 97 & Centrosema pubescens Benth. & $\begin{array}{l}\text { Projapoti } \\
\text { lata }\end{array}$ & Fabaceae & $\mathrm{D}$ & $\mathrm{CH}$ & Anwar-0305 \\
\hline 98 & Ceriscoides turgida (Roxb.) Tirveng. & Mota boilem & Rubiaceae & $\mathrm{D}$ & $\mathrm{T}$ & Anwar-0316 \\
\hline 99 & Cheilanthes belangeri (Bory) C.Chr. & Sada dhekia & Pteridaceae & $\mathrm{F}$ & $\mathrm{FH}$ & Anwar-0016 \\
\hline 100 & Chrinum asiaticum $\mathrm{L}$. & $\begin{array}{l}\text { Such } \\
\text { dadshon }\end{array}$ & Liliaceae & M & $\mathrm{H}$ & Anwar-1048 \\
\hline 101 & $\begin{array}{l}\text { Chromolaena odorata (L.) King \& } \\
\text { Robinson }\end{array}$ & German lata & Asteraceae & $\mathrm{D}$ & S & Anwar-0726 \\
\hline 102 & $\begin{array}{l}\text { Chrysalidocarpus lutescens (Bory) H. } \\
\text { Wendl. }\end{array}$ & Areca palm & Arecaceae & M & S & Anwar-0959 \\
\hline 103 & Cissampelos pareira $\mathrm{L}$. & Tubaki-lata & Menispermaceae & $\mathrm{D}$ & $\mathrm{CH}$ & Anwar-0766 \\
\hline 104 & Cissus adnata Roxb. & Bhatia-lata & Vitaceae & $\mathrm{D}$ & $\mathrm{CH}$ & Anwar-1072 \\
\hline 105 & Cissus assamica (Lawson) Craib & Assam lata & Vitaceae & $\mathrm{D}$ & $\mathrm{CH}$ & Anwar-1062 \\
\hline 106 & Cissus javana DC. & Dukhu lata & Vitaceae & $\mathrm{D}$ & $\mathrm{CH}$ & Anwar-0579 \\
\hline 107 & $\begin{array}{l}\text { Clausena suffruticosa (Roxb.) Wight \& } \\
\text { Arn. }\end{array}$ & Pan porag & Rutaceae & $\mathrm{D}$ & S & Anwar-0737 \\
\hline 108 & Cleome viscose $\mathrm{L}$. & Hurhurey & Capparaceae & $\mathrm{D}$ & $\mathrm{H}$ & Anwar-0260 \\
\hline 109 & Clerodendrum viscosum Pers. & Vat & Verbenaceae & $\mathrm{D}$ & S & Anwar-0736 \\
\hline 110 & Coccinia grandis (L.) Voigt & Telakucha & Cucurbitaceae & $\mathrm{D}$ & $\mathrm{CH}$ & Anwar-0896 \\
\hline 111 & Codiaeum variegatum (L.) A. Juss. & Patabahar & Euphorbiaceae & $\mathrm{D}$ & $\mathrm{S}$ & Anwar-1116 \\
\hline 112 & Coffea arabica $\mathrm{L}$. & Coffee & Rubiaceae & $\mathrm{D}$ & $\mathrm{S}$ & Anwar-1064 \\
\hline
\end{tabular}




\begin{tabular}{|c|c|c|c|c|c|c|}
\hline 113 & Coffea bengalensis Roxb. ex Schult. & Ban coffee & Rubiaceae & $\mathrm{D}$ & $\mathrm{S}$ & Anwar-0268 \\
\hline 114 & Colocasia esculenta (L.) Schott & Kachu & Araceae & M & $\mathrm{H}$ & Anwar-0889 \\
\hline 115 & Combretum acuminatum Roxb. & Patyuni & Combretaceae & $\mathrm{D}$ & S & Anwar-1119 \\
\hline 116 & Conyza floribunda Kunth & Not known & Asteraceae & $\mathrm{D}$ & $\mathrm{H}$ & Anwar-0038 \\
\hline 117 & Conyza semipinnatifida Wall. ex $\mathrm{DC}$. & $\begin{array}{l}\text { Adha } \\
\text { konyza }\end{array}$ & Asteraceae & $\mathrm{D}$ & $\mathrm{H}$ & Anwar-0598 \\
\hline 118 & Cordia dichotoma Forst. F. & Boula bahul & Boraginaceae & $\mathrm{D}$ & $\mathrm{T}$ & Anwar-0308 \\
\hline 119 & Costus speciosus (Koenig ex Retz.) Smith & Keomul & Costaceae & M & $\mathrm{H}$ & Anwar-1023 \\
\hline 120 & Crescentia cujete L. & Dugdugi & Bignoniaceae & $\mathrm{D}$ & ST & Anwar-1112 \\
\hline 121 & $\begin{array}{l}\text { Crotalaria acicularis Buch.-Ham. ex } \\
\text { Benth. \& Hook. }\end{array}$ & $\begin{array}{l}\text { Kata } \\
\text { Jhunjhuni }\end{array}$ & Fabaceae & $\mathrm{D}$ & $\mathrm{H}$ & Anwar-0061 \\
\hline 122 & Crotalaria pallida Ait. & Jhunjhuni & Fabaceae & $\mathrm{D}$ & $\mathrm{H}$ & Anwar-0193 \\
\hline 123 & Crotalaria sessiliflora $\mathrm{L}$. & $\begin{array}{l}\text { Silai } \\
\text { Jhunjhuni }\end{array}$ & Fabaceae & $\mathrm{D}$ & S & Anwar-1090 \\
\hline 124 & Crotalaria spectabilis Roth. & $\begin{array}{l}\text { Pipli- } \\
\text { Jhunjhun }\end{array}$ & Fabaceae & $\mathrm{D}$ & $\mathrm{H}$ & Anwar-0596 \\
\hline 125 & Croton bonplandianus Baill. & Bancroton & Euphorbiaceae & $\mathrm{D}$ & $\mathrm{H}$ & Anwar-0888 \\
\hline 126 & Croton caudatus Geisel. & Sabagjala & Euphorbiaceae & $\mathrm{D}$ & SS & Anwar-0626 \\
\hline 127 & Curculigo latifolia (Dryand.) Ait. & Talmuli & Liliaceae & M & $\mathrm{H}$ & Anwar-0630 \\
\hline 128 & Curcuma zedoaria (Christm.) Rose. & Shati & Zingiberaceae & M & $\mathrm{H}$ & Anwar-0920 \\
\hline 129 & Cuscuta reflexa Roxb. & Swarnolata & Cuscutaceae & $\mathrm{D}$ & $\mathrm{H}$ & Anwar-0900 \\
\hline 130 & Cycas pectinata Buch.-Ham. & Moniraj & Cycadaceae & $\mathrm{G}$ & ST & Anwar -0941 \\
\hline 131 & Cymbopogon citratus (DC.) Stapf & $\begin{array}{l}\text { Dhan } \\
\text { sabarang }\end{array}$ & Poaceae & M & $\mathrm{H}$ & Anwar-1053 \\
\hline 132 & Cynodon dactylon (L.) Pers. & Durba & $\mathrm{Po}$ & M & $\mathrm{H}$ & Anwar-0950 \\
\hline 133 & Cyperus cyperoides (L.) O. Ktze. & a & $\mathrm{Cy}$ & $\mathrm{M}$ & $\mathrm{H}$ & Anwar-0050 \\
\hline 134 & Cyperus distans L. f. & $\begin{array}{l}\text { Pani } \\
\text { Malanga }\end{array}$ & & M & $\mathrm{H}$ & Anwar-0421 \\
\hline 135 & Cyperus albostriatus Schrad. & Not known & $\mathrm{Cy}$ & M & $\mathrm{H}$ & r-1097 \\
\hline 136 & Cyperus rotundus Benth. & a/Vadla & Cyp & M & $\mathrm{H}$ & Anwar-1093 \\
\hline 137 & Cyrtococcum oxyphyllum (Steud.) Stapf & $\begin{array}{l}\text { Coccum } \\
\text { ghas }\end{array}$ & Poaceae & M & $\mathrm{H}$ & Anwar-1118 \\
\hline 138 & Dalbergia pinnata (Lour.) Prain & Chakemdia & $\mathrm{Fal}$ & $\mathrm{D}$ & SS & Anwar-1043 \\
\hline 139 & Dehaasia kurzii King ex Hook. f. & Madanmosto & Laur & $\mathrm{D}$ & $\mathrm{T}$ & Anwar-0679 \\
\hline 140 & Dillenia pentagyna Roxb. & Hargaza & Dille & $\mathrm{D}$ & $\mathrm{T}$ & Anwar-0947 \\
\hline 141 & Delonix regia Rafin. & $\begin{array}{l}\text { Krishnochur } \\
\text { a }\end{array}$ & Caesalpiniaceae & $\mathrm{D}$ & $\mathrm{T}$ & Anwar-0931 \\
\hline 142 & Dendrolobium triangulare (Retz.) Merr. & Bir-jarwar & Fabaceae & $\mathrm{D}$ & S & Anwar-1046 \\
\hline 143 & Dentella repens (L.) J. R. \& G. Forst & Bhuipat & Rubiaceae & $\mathrm{D}$ & $\mathrm{H}$ & Anwar-1096 \\
\hline 144 & Dentella serpyllifolia Wall. ex Craib & Bhuipat & Rubiaceae & $\mathrm{D}$ & $\mathrm{H}$ & Anwar-0965 \\
\hline 145 & Derris scandens (Roxb.) Benth. & Amkurchi & Fabaceae & $\mathrm{D}$ & $\mathrm{CS}$ & Anwar-0894 \\
\hline 146 & Desmodium heterophyllum (Willd.) DC. & $\begin{array}{l}\text { Bon- } \\
\text { motorsuti }\end{array}$ & Fabaceae & $\mathrm{D}$ & $\mathrm{H}$ & Anwar-1120 \\
\hline 147 & Desmodium triflorum (L.) DC. & Kodalia & & $\mathrm{D}$ & $\mathrm{H}$ & $r-0638$ \\
\hline 148 & Desmos chinensis Lour. & alang & & $\mathrm{D}$ & $\mathrm{S}$ & Anwar-0568 \\
\hline 149 & Desmos dumosus (Roxb.) Safford & Dumoyalang & Annonaceae & $\mathrm{D}$ & CS & Anwar-1106 \\
\hline 150 & Dichanthium caricosum (L.) A. Camus & Detara & Poaceae & M & $\mathrm{H}$ & Anwar-0597 \\
\hline 151 & Digitaria longiflora (Retz.) Pers. & $\begin{array}{l}\text { Choto fulka } \\
\text { ghas }\end{array}$ & Poaceae & M & $\mathrm{H}$ & Anwar-1108 \\
\hline 152 & Dillenia indica $\mathrm{L}$. & Chalta & & $\mathrm{D}$ & $\mathrm{T}$ & r-0884 \\
\hline 153 & Dillenia pentagyna Roxb. & Ajobi gach & Dille & $\mathrm{D}$ & $\mathrm{T}$ & Anwar-0935 \\
\hline 154 & Dioscorea pentaphylla L. & Kanta alu & Dioscoreaceae & M & $\mathrm{CH}$ & Anwar-0013 \\
\hline 155 & Diospyros blancoi A. DC. & Bon gub & Ebenaceae & $\mathrm{D}$ & $\mathrm{T}$ & Anwar-1079 \\
\hline 156 & Diospyros malabarica (Desr.) Kostel. & Deshi gab & Ebnaceae & $\mathrm{D}$ & $\mathrm{T}$ & Anwar-0886 \\
\hline 157 & Dracaena spicata Roxb. & Gandhok & Agavaceae & M & $\mathrm{S}$ & Anwar-0631 \\
\hline
\end{tabular}




\begin{tabular}{|c|c|c|c|c|c|c|}
\hline 158 & Drynaria quercifolia (L.) J. Sm. & Chile fern & Polypodiaceae & $\mathrm{F}$ & FH & Anwar-0295 \\
\hline 159 & Duranta repens L. & Kata mehedi & Verbenaceae & $\mathrm{D}$ & S & Anwar-0628 \\
\hline 160 & Dysoxylum excelsum Blume, Bijdr & Lambu & Meliaceae & $\mathrm{D}$ & $\mathrm{T}$ & Anwar-0994 \\
\hline 161 & Eclipta alba (L.) Hassk. & Kalo keshi & Asteraceae & $\mathrm{D}$ & $\mathrm{H}$ & Anwar-0809 \\
\hline 162 & Elatostema papillosum Wedd. & Sila jhara & Urticaceae & $\mathrm{D}$ & $\mathrm{H}$ & Anwar-1083 \\
\hline 163 & Elatostema sesquifolium (Blume) Hassk. & Seskijhara & Urticaceae & $\mathrm{D}$ & $\mathrm{H}$ & Anwar-0423 \\
\hline 164 & Elaeocarpus floribundus Blume & Jolpie & Eleocarpaceae & $\mathrm{D}$ & $\mathrm{T}$ & Anwar-0938 \\
\hline 165 & Eleusine indica (L.) Gaertn. & $\begin{array}{l}\text { Ghora } \\
\text { dubboher }\end{array}$ & Poaceae & M & $\mathrm{H}$ & Anwar-0854 \\
\hline 166 & Eragrostis tenella $(\mathrm{L}$.$) P. Beauv$ & Koni ghas & Poaceae & M & $\mathrm{H}$ & Anwar-0197 \\
\hline 167 & Eranthemum album Nees & $\begin{array}{l}\text { Madhuban } \\
\text { sak }\end{array}$ & Acantheceae & $\mathrm{D}$ & $\mathrm{H}$ & Anwar-0583 \\
\hline 168 & Eriochloa procera (Retz.) C. E. Hubb. & Nalghas & Poaceae & M & $\mathrm{H}$ & Anwar-0415 \\
\hline 169 & Eucalyptus camaldulensis Dehnhardt & Eucalyptus & Myrtaceae & $\mathrm{D}$ & $\mathrm{T}$ & Anwar-0914 \\
\hline 170 & Eulalia fastigiata (Nees ex Steud.) Haines & $\begin{array}{l}\text { Eulalifasti } \\
\text { ghas }\end{array}$ & Poaceae & M & $\mathrm{H}$ & Anwar-0413 \\
\hline 171 & Euphorbia antiquorum $\mathrm{L}$. & Kata gachh & Euphorbiaceae & $\mathrm{D}$ & ST & Anwar-1069 \\
\hline 172 & Euphorbia hirta L. & Dudhia & Euphorbiaceae & $\mathrm{D}$ & $\mathrm{H}$ & Anwar-0202 \\
\hline 173 & Euphorbia thymifolia L. & Dudhiya & Euphorbiaceae & $\mathrm{D}$ & $\mathrm{H}$ & Anwar-0881 \\
\hline 174 & Evolvulus nummularius (L.) L. & Khetpapri & Convolvulaceae & $\mathrm{D}$ & $\mathrm{H}$ & Anwar-1051 \\
\hline 175 & Ficus hispida (FH) Linn. & Kakdumur & Moraceae & $\mathrm{D}$ & ST & Anwar-0722 \\
\hline 176 & Ficus religiosa $\mathrm{L}$. & Ashwath & Moraceae & $\mathrm{D}$ & $\mathrm{T}$ & Anwar-1068 \\
\hline 177 & Ficus benghalensis L. & Bot & Moraceae & $\mathrm{D}$ & $\mathrm{T}$ & Anwar-1008 \\
\hline 178 & Ficus lyrata Warb. & Lyrate bat & Moraceae & $\mathrm{D}$ & $\mathrm{T}$ & Anwar-1099 \\
\hline 179 & cemosa L. & Jagyadumur & Moraceae & $\mathrm{D}$ & $\mathrm{T}$ & Anwar-1127 \\
\hline 180 & Ficus & 0 & Morac & $\mathrm{D}$ & $\mathrm{T}$ & Anwar-0018 \\
\hline 181 & Flacourtia indica (Burm. f.) Merr. & ii & aceae & $\mathrm{D}$ & S & Anwar-1078 \\
\hline 182 & gomas ( & & $\mathrm{F}$ & $\mathrm{D}$ & ST & -0668 \\
\hline 183 & Flemingias & usak & & $\mathrm{D}$ & $\mathrm{H}$ & $r-0516$ \\
\hline 184 & Flueggea virosa (Roxb. ex Willd.) Baill. & Shikori & Eup & $\mathrm{D}$ & $\mathrm{S}$ & $r-0106$ \\
\hline 185 & Garcinia cowa Roxb. & Kao & $\mathrm{Clu}$ & $\mathrm{D}$ & $\mathrm{T}$ & -0997 \\
\hline 186 & Garcinia morella $(\mathrm{Ga}$ & al & & $\mathrm{D}$ & $\mathrm{T}$ & Anwar-0960 \\
\hline 187 & Gardenia coronaria Bueh.-Ham. & ramal & $\mathrm{Ru}$ & $\mathrm{D}$ & $\mathrm{T}$ & Anwar-1055 \\
\hline 188 & Globba multiflora Wall. ex Baker & $\begin{array}{l}\text { Shukh } \\
\text { globba }\end{array}$ & Zingiberaceae & M & $\mathrm{H}$ & Anwar-1066 \\
\hline 189 & Glycosmis pentaphylla (Retz.) A. DC. & Motkila & Ruta & $\mathrm{D}$ & S & Anwar-0613 \\
\hline 190 & Grewia asiatica L. & Falsa & Tilia & $\mathrm{D}$ & $\mathrm{T}$ & Anwar-0034 \\
\hline 191 & Grewia nervosa (Lour.) Panigr. & Datoi & Tiliaceae & $\mathrm{D}$ & ST & Anwar-0754 \\
\hline 192 & Grewia tilliaefolia Vahl & Pholsa & Tiliaceae & $\mathrm{D}$ & ST & Anwar-0979 \\
\hline 193 & Hedyotis corymbosa (L.) Lamk. & Khet papra & Rubiaceae & $\mathrm{D}$ & $\mathrm{H}$ & Anwar-1060 \\
\hline 194 & Heliotropium indicum $\mathrm{L}$. & Hatishur & Boraginaceae & $\mathrm{D}$ & $\mathrm{H}$ & Anwar-0915 \\
\hline 195 & Hemidesmus indicus (L.) R. Br. & Anantomul & Asclepiadaceae & $\mathrm{D}$ & $\mathrm{CH}$ & Anwar-0735 \\
\hline 196 & $\begin{array}{l}\text { Hevea brasiliensis (Willd. ex A. Juss.) } \\
\text { Muell.-Arg. }\end{array}$ & Rubber & Euphorbiaceae & $\mathrm{D}$ & $\mathrm{T}$ & Anwar-0904 \\
\hline 197 & Hibiscus surattensis L. & & & $\mathrm{D}$ & $\mathrm{CH}$ & $r-0597$ \\
\hline 198 & rocarpa (Blume) Cogn. & $\mathrm{Gu}$ & itaceae & $\mathrm{D}$ & $\mathrm{CH}$ & Anwar-0672 \\
\hline 199 & Holarrhena antidysenterica (Linn.)Wall. & Kurchi & naceae & $\mathrm{D}$ & ST & Anwar-0633 \\
\hline 200 & Hydnocarpus kurzii (King) Warb & Chaolmugra & iaceae & $\mathrm{D}$ & $\mathrm{ST}$ & Anwar-0987 \\
\hline 201 & cemosum (Roxb.) & Nar & ceae & $\mathrm{D}$ & S & ar-1107 \\
\hline 202 & Hyptis suaveolens (L.) Poit. & Tokma & aceae & $\mathrm{D}$ & VH & Anwar-0590 \\
\hline 203 & Ichnocarpus frutescens $\mathrm{R}$. Br. & Dudhila & Apocynacea & $\mathrm{D}$ & $\mathrm{CH}$ & Anwar-0788 \\
\hline 204 & Ipomoea fistulosa Mart. ex Choisy & Dholkalmi & Convolvulaceae & $\mathrm{D}$ & $S$ & Anwar-0952 \\
\hline 205 & Ipomoea hederifolia $\mathrm{L}$. & Lal-kalmi & Convolvulaceae & $\mathrm{D}$ & $\mathrm{CH}$ & Anwar-0349 \\
\hline 206 & Ipomoea mauritiana Jacq. & Kolmi & Convolvulaceae & $\mathrm{D}$ & $\mathrm{H}$ & Anwar-0879 \\
\hline 207 & Ixora acuminata Roxb. & Nata rangan & Rubiaceae & $\mathrm{D}$ & $\mathrm{S}$ & Anwar-0420 \\
\hline
\end{tabular}




\begin{tabular}{|c|c|c|c|c|c|c|}
\hline 208 & Ixora cuneifolia Roxb. & Ban rangon & Rubiaceae & $\mathrm{D}$ & $\mathrm{S}$ & Anwar-0721 \\
\hline 209 & Jasminum sambac (L.) Ait. & Beli & Oleaceae & $\mathrm{D}$ & S & Anwar-0640 \\
\hline 210 & Jatropha glandulifera Roxb. & Lal verenda & Euphorbiaceae & $\mathrm{D}$ & S & Anwar- 0870 \\
\hline 211 & Justicia gendarussa Burm. f. & Bish-jarul & Acantheceae & $\mathrm{D}$ & S & Anwar-0910 \\
\hline 212 & Kopsia fruticosa (Ker.) A. DC & Dakur & Apocynaceae & $\mathrm{D}$ & $\mathrm{S}$ & Anwar-0129 \\
\hline 213 & $\begin{array}{l}\text { Kyllinga nemoralis (J. R. Forst. \& G } \\
\text { Forst.) Dandy }\end{array}$ & $\begin{array}{l}\text { Subasinir } \\
\text { bishi }\end{array}$ & Cyperaceae & $\mathrm{M}$ & $\mathrm{H}$ & Anwar-0916 \\
\hline 214 & Lagerstroemia indica $\mathrm{L}$. & Choto jarul & Lythraceae & $\mathrm{D}$ & $\mathrm{T}$ & Anwar-0885 \\
\hline 215 & Lagerstroemia speciosa (L.) Pers & Jarul & Lythraceae & $\mathrm{D}$ & $\mathrm{T}$ & Anwar-0313 \\
\hline 216 & Lannea coromandelica (Houtt.) Merr. & Jiga & Anacardiaceae & $\mathrm{D}$ & $\mathrm{T}$ & Anwar-0993 \\
\hline 217 & Lantana camara $\mathrm{L}$. & Kutush kata & Verbenaceae & $\mathrm{D}$ & $\mathrm{S}$ & Anwar-0612 \\
\hline $\begin{array}{c}21 \\
8\end{array}$ & Laportea interrupta (L.) Chew & Chutra & Urticaceae & $\mathrm{D}$ & $\mathrm{H}$ & Anwar-0573 \\
\hline 219 & Leea rubra Royle & Lal leea & Leeaceae & $\mathrm{D}$ & $\mathrm{H}$ & Anwar-0637 \\
\hline 220 & Lepisanthes senegalensis (Poir.) Leenh. & Sagol ladi & Sapindaceae & $\mathrm{D}$ & ST & Anwar-0760 \\
\hline 221 & Leucaena leucocephala (Lamk.) de Wit. & Ipil ipil & Mimosaceae & $\mathrm{D}$ & $\mathrm{T}$ & Anwar-0706 \\
\hline 222 & Leucas aspera (Willd.) Link & Sheto drawn & Lamiaceae & $\mathrm{D}$ & $\mathrm{H}$ & Anwar-0190 \\
\hline 223 & $\begin{array}{l}\text { Lindenbergia muraria (Roxb. ex D. Don) } \\
\text { P. }\end{array}$ & Bashanti & Scrophulariaceae & $\mathrm{D}$ & $\mathrm{H}$ & Anwar-0601 \\
\hline 224 & Lindernia multiflora (Roxb.) & Muli chapra & Scrophulariaceae & $\mathrm{D}$ & $\mathrm{H}$ & Anwar-0906 \\
\hline 225 & Lindernia procumbens (Krocker) Philcox & Bakpuspa & Scrophulariaceae & $\mathrm{D}$ & $\mathrm{H}$ & Anwar-0999 \\
\hline 226 & Lindernia antipoda (L.) Alston & Zaighas & Scrophulariaceae & $\mathrm{D}$ & $\mathrm{H}$ & Anwar-0985 \\
\hline 227 & Lindernia ciliata (Colsra.) Pennell & Bhui & Scrophulariaceae & $\mathrm{D}$ & $\mathrm{H}$ & Anwar-0874 \\
\hline 228 & Lindernia crustacea (L.) F. Muell. & Chapraghas & Scrophulariaceae & $\mathrm{D}$ & $\mathrm{H}$ & Anwar-0998 \\
\hline 229 & Lindernia hyssopioides (L.) Haines & Sopi chapra & Scrophulariaceae & $\mathrm{D}$ & $\mathrm{H}$ & Anwar-0917 \\
\hline 230 & Litchi chinensis Sonn. & Lechu & Sapindaceae & $\mathrm{D}$ & $\mathrm{T}$ & Anwar-0990 \\
\hline 231 & Litsea glutinosa (Lour.) Robinson & Kukur chita & Lauraceae & $\mathrm{D}$ & $\mathrm{T}$ & Anwar-0078 \\
\hline 232 & Litsea monopetala (Roxb.) Pers. & Pipulti & Lauraceae & $\mathrm{D}$ & $\mathrm{T}$ & Anwar- 0750 \\
\hline 233 & Lygodium flexuosum (Linn) Sw. & Lata dhekia & Lygodiaceae & $\mathrm{F}$ & $\mathrm{CH}$ & Anwar-0141 \\
\hline 234 & $\begin{array}{l}\text { Lygodium giganteum Tagawa \& } \mathrm{K} \text {. } \\
\text { Iwatsuki }\end{array}$ & Lata dhekia & Lygodiaceae & $\mathrm{F}$ & $\mathrm{CH}$ & Anwar-0869 \\
\hline 235 & Lygodium japonicum (Thunb.) Sw & Lata dhekia & Lygodiaceae & $\mathrm{F}$ & $\mathrm{CH}$ & Anwar-0976 \\
\hline 236 & Macaranga indica Wight & Gulli & Euphorbiaceae & $\mathrm{D}$ & ST & Anwar-0112 \\
\hline 237 & Madhuca longifolia (Koenig) MacBride & Mahua & Sapotaceae & $\mathrm{D}$ & $\mathrm{T}$ & Anwar-0983 \\
\hline 238 & Maesa chisia F. Ham. ex D. Don & Gangu-loda & Myrsinaceae & $\mathrm{D}$ & VS & Anwar-0753 \\
\hline 239 & Mallotus tetracoccus (Roxb.) Kurz. & Kumaribura & Euphorbiaceae & $\mathrm{D}$ & $\mathrm{T}$ & Anwar-0275 \\
\hline 240 & Mallotus philippensis (Lamk.) Muell.-Arg. & Sinduri & Euphorbiaceae & $\mathrm{D}$ & ST & Anwar-0684 \\
\hline 241 & Mallotus repandus (Willd.) Muell.-Arg. & Chot sinduri & Euphorbiaceae & $\mathrm{D}$ & SS & Anwar-0871 \\
\hline 242 & Mangifera indica $\mathrm{L}$. & $\mathrm{Am}$ & Anacardiaceae & $\mathrm{D}$ & $\mathrm{T}$ & Anwar-0996 \\
\hline 243 & Mangifera sylvatica Roxb. & Uri am & Anacardiaceae & $\mathrm{D}$ & $\mathrm{T}$ & Anwar-0883 \\
\hline 244 & Manihot esculenta Crantz & Kasava & Euphorbiaceae & $\mathrm{D}$ & $S$ & Anwar-0127 \\
\hline 245 & Marsilea minuta $\mathrm{L}$. & Sunsi shak & Marsileaceae & $\mathrm{F}$ & $\mathrm{FH}$ & Anwar-0882 \\
\hline 246 & Mecardonia procumbens (Mill.) Small & Mikardan & Scrophulariaceae & $\mathrm{D}$ & $\mathrm{H}$ & Anwar-0885 \\
\hline 247 & Melastoma malabathricum $\mathrm{L}$. & Dat ranga & Melastomaceae & $\mathrm{D}$ & S & Anwar-0319 \\
\hline 248 & Melia azedarach $\mathrm{L}$. & Ghoranim & Meliaceae & $\mathrm{D}$ & $\mathrm{T}$ & Anwar-0908 \\
\hline 249 & Melocanna baccifera (Roxb.) Kurz & Muli & Poaceae & $\mathrm{D}$ & SS & Anwar-0961 \\
\hline 250 & Melochia corchorifolia $\mathrm{L}$. & Ban pat & Sterculiaceae & $\mathrm{D}$ & $\mathrm{H}$ & Anwar-0808 \\
\hline 251 & Merremia vitifolia (Burm. f.) Hallier f. & Kargolata & Convolvulaceae & $\mathrm{D}$ & $\mathrm{CH}$ & Anwar-0657 \\
\hline 252 & Mesua ferrea $\mathrm{L}$. & Nagessor & Clusiaceae & $\mathrm{D}$ & ST & Anwar-0227 \\
\hline 253 & $\begin{array}{l}\text { Micromelum minutum (G. Forster) Wight } \\
\& \text { Arn. }\end{array}$ & Bankunch & Rutaceae & $\mathrm{D}$ & ST & Anwar-0956 \\
\hline 254 & Mikania cordata (Burm. f.) Robinson & Jessri lata & Asteraceae & $\mathrm{D}$ & $\mathrm{CH}$ & Anwar-0730 \\
\hline 255 & Mimosa diplotricha C. Wright ex Sauvalle & $\begin{array}{l}\text { Borolazzabo } \\
\text { ti }\end{array}$ & Mimosaceae & $\mathrm{D}$ & $\mathrm{H}$ & Anwar-0594 \\
\hline 256 & Mimosa pudica L. & Lozzaboti & Mimosaceae & $\mathrm{D}$ & $\mathrm{H}$ & Anwar-0972 \\
\hline
\end{tabular}




\begin{tabular}{|c|c|c|c|c|c|c|}
\hline 257 & Mimusops elengi L. & Bakul & Sapotaceae & $\mathrm{D}$ & $\mathrm{T}$ & Anwar-0930 \\
\hline 258 & Mitracarpus hirtus (L.) DC. & Tupi kadam & Rubiaceae & $\mathrm{D}$ & $\mathrm{H}$ & Anwar-0281 \\
\hline 259 & Mitragyna parvifolia (Roxb.) Korth. & Phuti kadam & Rubiaceae & $\mathrm{D}$ & $\mathrm{T}$ & Anwar-1001 \\
\hline 260 & Modecca trilobata Roxb. & Rakhal sasha & Passifloraceae & $\mathrm{D}$ & $\mathrm{CH}$ & Anwar-0667 \\
\hline 261 & Morinda angustifolia Roxb. & Daru horidra & Rubiaceae & $\mathrm{D}$ & $\mathrm{S}$ & Anwar-0099 \\
\hline 262 & Mucuna pruriens (L.) DC. & Khamach & Fabaceae & $\mathrm{D}$ & VH & Anwar-1032 \\
\hline 263 & Muntingia calabura $\mathrm{L}$. & China clesri & Tiliaceae & $\mathrm{D}$ & ST & Anwar-1109 \\
\hline 264 & Murraya paniculata (L.) Jack & Kamini & Rutaceae & $\mathrm{D}$ & ST & Anwar-0139 \\
\hline 265 & Musa acuminata Colla & Pahari kola & Musaceae & M & $\mathrm{H}$ & Anwar-0924 \\
\hline 266 & Mussaenda glabra Vahl & Patra lekha & Rubiaceae & $\mathrm{D}$ & $\mathrm{S}$ & Anwar-0957 \\
\hline 267 & Mussaenda roxburghii Hooker f. & $\begin{array}{l}\text { Chauri- } \\
\text { chaonri }\end{array}$ & Rubiaceae & $\mathrm{D}$ & $\mathrm{S}$ & Anwar-1005 \\
\hline 268 & Mussaenda frondosa $\mathrm{L}$. & $\begin{array}{l}\text { Ban } \\
\text { mussaenda }\end{array}$ & Rubiaceae & $\mathrm{D}$ & $\mathrm{S}$ & Anwar-0986 \\
\hline 269 & Mycetia longifolia (Wall.) K. Schum. & Mycetelon & Rubiaceae & $\mathrm{D}$ & S & Anwar-0620 \\
\hline 270 & Myхоругum smilacifolium (Wall.) Blume & Panta lata & Oleaceae & $\mathrm{D}$ & $\mathrm{CS}$ & Anwar-0007 \\
\hline 271 & Nelsonia canescens (Lamk.) Spreng. & Paramul & Acantheceae & $\mathrm{D}$ & $\mathrm{S}$ & Anwar-1041 \\
\hline 272 & Neolamarckia cadamba (Roxb.) Bosser & Kodom & Rubiaceae & $\mathrm{D}$ & $\mathrm{T}$ & Anwar-0926 \\
\hline 273 & Nyctanthes arbor-tristis L. & Sheuli & Verbenaceae & $\mathrm{D}$ & ST & Anwar-0571 \\
\hline 274 & Ocimum americanum $\mathrm{L}$. & Bantulsi & Lamiaceae & $\mathrm{D}$ & $S$ & Anwar-0892 \\
\hline 275 & Ocimum sanctum $\mathrm{L}$. & Tulsi & Lamiaceae & $\mathrm{D}$ & S & Anwar-1035 \\
\hline 276 & Olea dioica Roxb. & Atajam & Oleaceae & $\mathrm{D}$ & $\mathrm{T}$ & Anwar-0954 \\
\hline 277 & Operculina turpethum (L.) S. Manso & Teurimul & Convolvulaceae & $\mathrm{D}$ & $\mathrm{CH}$ & Anwar-0513 \\
\hline 278 & Ophiorrhiza mungos $\mathrm{L}$. & Kalashona & Rubiaceae & $\mathrm{D}$ & S & Anwar-1011 \\
\hline 279 & Oroxylum indicum (L.) Kurz & Sona dinga & Bignoniaceae & $\mathrm{D}$ & $\mathrm{T}$ & Anwar-0929 \\
\hline 280 & Oxalis corniculata $\mathrm{L}$. & Amrul shak & Oxalidaceae & $\mathrm{D}$ & $\mathrm{H}$ & Anwar-0469 \\
\hline 281 & Oxalis corymbosa DC. & $\begin{array}{l}\text { Golapi } \\
\text { amrul }\end{array}$ & Oxalidaceae & $\mathrm{D}$ & $\mathrm{H}$ & Anwar-0880 \\
\hline 282 & Pedilanthus tithymaloides Poit. & Rangchita & Euphorbiaceae & $\mathrm{D}$ & S & Anwar-1022 \\
\hline 283 & Paederia foetida $\mathrm{L}$. & $\begin{array}{l}\text { Gondhovedu } \\
\text { li }\end{array}$ & Rubiaceae & $\mathrm{D}$ & $\mathrm{CH}$ & Anwar-0187 \\
\hline 284 & Pandanus fascicularis Lamk. & Keya & Pandanaceae & M & S & Anwar-0953 \\
\hline 285 & Panicum maximum Jacq & Gini ghas & Poaceae & M & $\mathrm{H}$ & Anwar-1039 \\
\hline 286 & Paspalidium flavidum (Retz.) A. Camus & Bolai mandi & Poaceae & M & $\mathrm{H}$ & Anwar-0060 \\
\hline 287 & Paspalum longifolium Roxb. & Lamtafuli & Poaceae & M & $\mathrm{H}$ & Anwar-0673 \\
\hline 288 & Passiflora foetida $\mathrm{L}$. & Jhumka lata & Passifloraceae & $\mathrm{D}$ & $\mathrm{CH}$ & Anwar-0272 \\
\hline 289 & Pavetta assamica Bremek & $\begin{array}{l}\text { Assami } \\
\text { bonomali }\end{array}$ & Rubiaceae & $\mathrm{D}$ & $S$ & Anwar-0878 \\
\hline 290 & Pavetta indica $\mathrm{L}$. & Banamali & Rubiaceae & $\mathrm{D}$ & S & Anwar-0711 \\
\hline 291 & Peliosanthes teta Andr. & Napigach & Haemoderaceae & M & $\mathrm{H}$ & Anwar-0023 \\
\hline 292 & $\begin{array}{l}\text { Peltophorum pterocarpum (DC.) K. } \\
\text { Heyrie }\end{array}$ & $\begin{array}{l}\text { Halud } \\
\text { krishnochura }\end{array}$ & Caesalpiniaceae & $\mathrm{D}$ & $\mathrm{T}$ & Anwar-0728 \\
\hline 293 & Peperomia pellucida (L.) H. B. \& K. & Luchi pata & Peperomiaceae & $\mathrm{D}$ & $\mathrm{H}$ & Anwar-0912 \\
\hline 294 & Pericampylus glaucus ( Lamk.) Merr. & Goria loti & $\begin{array}{l}\text { Menispermacea } \\
\mathrm{e}\end{array}$ & $\mathrm{D}$ & $\mathrm{CH}$ & Anwar-0746 \\
\hline 295 & Persicaria flaccida (Meissn.) H. Gross & $\begin{array}{l}\text { Lata } \\
\text { bashkatali }\end{array}$ & Polygonaceae & $\mathrm{D}$ & $\mathrm{H}$ & Anwar-0615 \\
\hline 296 & Phaulopsis imbricate (Forssk.) Sweet & Kantasi & Acanthaceae & $\mathrm{D}$ & $\mathrm{H}$ & Anwar-0762 \\
\hline 297 & Phoenix sylvestris Roxb. & $\begin{array}{l}\text { Deshi } \\
\text { Khejur }\end{array}$ & Arecaceae & M & $\mathrm{T}$ & Anwar-1017 \\
\hline 298 & Phyllanthus emblica $\mathrm{L}$. & Amlaki & Euphorbiaceae & $\mathrm{D}$ & $\mathrm{T}$ & Anwar-0732 \\
\hline 299 & Phyllanthus niruri $\mathrm{L}$. & Bhui amla & Euphorbiaceae & $\mathrm{D}$ & $\mathrm{H}$ & Anwar-1030 \\
\hline 300 & Phyllanthus reticulatus Poir. & Sitka & Euphorbiaceae & $\mathrm{D}$ & $\mathrm{S}$ & Anwar-0716 \\
\hline 301 & Physalis minima $\mathrm{L}$. & Phutka & Solanaceae & $\mathrm{D}$ & $\mathrm{H}$ & Anwar- 0320 \\
\hline 302 & Pilea melastomoides (Poir.) Wedd. & Mela & Urticaceae & $\mathrm{D}$ & $\mathrm{H}$ & Anwar-0940 \\
\hline
\end{tabular}




\begin{tabular}{|c|c|c|c|c|c|c|}
\hline 303 & Pinus kesiya Royle ex Grodon & $\begin{array}{l}\text { moricha } \\
\text { saral }\end{array}$ & Pinaceae & $\mathrm{G}$ & $\mathrm{T}$ & Anwar-0990 \\
\hline 304 & Piper peepuloides Roxb. & Pipal & Piperaceae & $\mathrm{D}$ & $\mathrm{H}$ & Anwar-0052 \\
\hline 305 & Piper sylvestre Lamk. & Ban pipul & Piperaceae & $\mathrm{D}$ & $\mathrm{CH}$ & Anwar-1026 \\
\hline 306 & Plumeria rubra $\mathrm{L}$. & Champa & Apocynaceae & $\mathrm{D}$ & ST & Anwar-1002 \\
\hline 307 & Pogonatherum crinitum (Thunb.) Kunth & Nitu banch & Poaceae & $\mathrm{M}$ & $\mathrm{H}$ & Anwar-0024 \\
\hline 308 & Polyalthia longifolia (Sonn.) Thw & Debdaru & Annonacea & $\mathrm{D}$ & $\mathrm{T}$ & Anwar-0923 \\
\hline 309 & Pongamia pinnata (L.) Pierre & Karamcha & Fabaceae & $\mathrm{D}$ & $\mathrm{T}$ & Anwar-0135 \\
\hline 310 & Portulaca grandiflora Hook. & Time ful & Portulacaceae & $\mathrm{D}$ & $\mathrm{H}$ & Anwar-0925 \\
\hline 311 & Pothos scandens L. & Kala-lata & Araceae & M & $\mathrm{CH}$ & Anwar-1036 \\
\hline 312 & Premna herbacea Roxb. & Bhuijam & Verbenaceae & $\mathrm{D}$ & $\mathrm{H}$ & Anwar-1000 \\
\hline 313 & Protium serratum (Wall. ex Coelbr.) Engl. & Gutgutya & Bursseraceae & $\mathrm{D}$ & $\mathrm{T}$ & Anwar-0887 \\
\hline 314 & Psidium guajava $\mathrm{L}$. & Peyara & Myrtaceae & $\mathrm{D}$ & $\mathrm{T}$ & Anwar-1014 \\
\hline 315 & Pteris longifolia $\mathrm{L}$. & Dhekia & Pteridaceae & $\mathrm{F}$ & $\mathrm{FH}$ & Anwar-0216 \\
\hline 316 & Pteris vitifolia $\mathrm{L}$. & Dhekia & Acrostichaceae & $\mathrm{F}$ & $\mathrm{FH}$ & Anwar-0932 \\
\hline 317 & $\begin{array}{l}\text { Pterospermum semisagittatum Buch.- } \\
\text { Ham. ex Roxb. }\end{array}$ & Bara Assar & Sterculiaceae & $\mathrm{D}$ & $\mathrm{T}$ & Anwar-0158 \\
\hline 318 & Putranjiva roxburghii Wall. & Jiapura & Euphorbiace & $\mathrm{D}$ & $\mathrm{T}$ & Anwar-0126 \\
\hline 319 & Randia longiflora Lamk. & Kutmal & Rubiaceae & $\mathrm{D}$ & S & Anwar-0922 \\
\hline 320 & Rauvolfia serpentina (L.) Benth. ex Kurz & $\begin{array}{l}\text { Swarpo } \\
\text { gondha }\end{array}$ & Apoc & $\mathrm{D}$ & $\mathrm{H}$ & r-0639 \\
\hline 321 & Rhaphidophora hookeri Schott & Taka lata & Araceae & M & $\mathrm{CH}$ & -1040 \\
\hline 322 & Ricinus communis $\mathrm{L}$. & Reri & Euphorbiaceae & $\mathrm{D}$ & $\mathrm{S}$ & -0905 \\
\hline 323 & $\begin{array}{l}\text { Rottboellia cochinchinensis (Lour.) W. } \\
\text { D.Clayton }\end{array}$ & Bara swati & Poaceae & M & $\mathrm{H}$ & Anw \\
\hline 324 & Rungia pectinata (L.) Nees. & & & $\mathrm{D}$ & $\mathrm{H}$ & \\
\hline 325 & Saccharum & $\mathrm{Te}$ & $\mathrm{Po}$ & M & $\mathrm{H}$ & 893 \\
\hline 326 & Sacrochlamys pulche & icha & & $\mathrm{D}$ & $S$ & 0045 \\
\hline 327 & Santalum album $\mathrm{L}$. & $\begin{array}{l}\text { Chandan } \\
\text { kath }\end{array}$ & Santalaceae & $\mathrm{D}$ & $\mathrm{T}$ & Anwar-1042 \\
\hline 328 & Saraca asoca (Roxb.) de Wild & Ashok & jiniaceae & $\mathrm{D}$ & ST & Anwa \\
\hline 329 & Sauropus androgynus (L.) Merr. & Mitha patri & Euphorbiaceae & $\mathrm{D}$ & $\mathrm{S}$ & Anwar-0021 \\
\hline 330 & Schefflera bengalensis Gamble & Not known & Araliaceae & $\mathrm{D}$ & $\mathrm{CH}$ & Anwar-1019 \\
\hline 331 & Schefflera elliptica (Blume) Harms & Jeng jil & Araliaceae & $\mathrm{D}$ & $S$ & Anwar-0046 \\
\hline 332 & Scindapsus officinalis & Gaj-pipul & Araceae & M & $\mathrm{CH}$ & Anwar-0221 \\
\hline 333 & Scleria terrestris (L.) Fassett & Dharal ghasi & Cyperaceae & M & $\mathrm{H}$ & Anwar-0964 \\
\hline 334 & Scoparia dulcis L. & Mushri dana $S$ & crophulariaceae & $\mathrm{D}$ & $\mathrm{H}$ & Anwar-0846 \\
\hline 335 & Scurrula parasitica $\mathrm{L}$. & $\begin{array}{l}\text { Parash } \\
\text { pargacha }\end{array}$ & Loranthaceae & $\mathrm{D}$ & $S$ & Anwar-0928 \\
\hline 336 & Senn & Badarlati & & $\mathrm{D}$ & ST & 328 \\
\hline 337 & Senna sophera (L.) Roxb. & Kolkasundha & Caesalpiniaceae & $\mathrm{D}$ & $\mathrm{H}$ & Anwar-0655 \\
\hline 338 & Senna tora $(\mathrm{L}$.$) Roxb.$ & $\begin{array}{l}\text { Choto } \\
\text { kolkasundhe }\end{array}$ & Caesalpiniaceae & $\mathrm{D}$ & $\mathrm{H}$ & Anwar-0831 \\
\hline 339 & .) Roxb. & Dadmordan & $\mathrm{Ca}$ & $\mathrm{D}$ & $S$ & 909 \\
\hline 340 & Senn & & & $\mathrm{D}$ & $\mathrm{T}$ & 951 \\
\hline 341 & mifolia (Koen.) Stapf & $\mathrm{Ur}$ & & M & $\mathrm{H}$ & 838 \\
\hline 342 & Setaria pumila (Poir.) Roem. \& Schult. & $\begin{array}{l}\mathrm{Hc} \\
\mathrm{ka}\end{array}$ & $\mathrm{Po}$ & M & $\mathrm{H}$ & -0982 \\
\hline 343 & $\begin{array}{l}\text { Setaria sphacelata (Schum.) Stapf \& C. E. } \\
\text { Hubb. }\end{array}$ & $\begin{array}{l}\text { Motapata } \\
\text { kawn }\end{array}$ & $\mathrm{P}$ & M & $\mathrm{H}$ & 046 \\
\hline 344 & Smilax macrophylla Roxb. & Kumarica & & 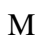 & $\mathrm{CH}$ & An \\
\hline 345 & Smilax ovalifolia Roxb. & Kumari kata & Smilaceae & M & $\mathrm{CH}$ & Anwar-0293 \\
\hline 346 & Solanum nigrum $\mathrm{L}$. & Tit begun & Solanaceae & $\mathrm{D}$ & $\mathrm{H}$ & Anwar-0863 \\
\hline 347 & Solanum torvum Swartz & Gothbegun & Solanaceae & $\mathrm{D}$ & $\mathrm{H}$ & Anwar-0434 \\
\hline 348 & Solena amplexicaulis (Lamk.) Gandhi & Rakhal sasha & Cucurbitaceae & $\mathrm{D}$ & $\mathrm{CH}$ & Anwar-0674 \\
\hline
\end{tabular}




\begin{tabular}{|c|c|c|c|c|c|c|}
\hline 349 & Spatholobus acuminatus Benth. & Palashi lata & Fabaceae & $\mathrm{D}$ & $\mathrm{CS}$ & Anwar-0329 \\
\hline 350 & Spermacoce latifolia Aublet & Ghuiojhi & Rubiaceae & $\mathrm{D}$ & $\mathrm{H}$ & Anwar-1006 \\
\hline 351 & Spilanthes calva DC. & $\begin{array}{l}\text { Surja } \\
\text { konnya }\end{array}$ & Asteraceae & $\mathrm{D}$ & $\mathrm{H}$ & Anwar-0962 \\
\hline 352 & Spondias pinnata (L. f.) Kurz & Amra & Anacardiaceae & $\mathrm{D}$ & $\mathrm{T}$ & Anwar-1031 \\
\hline 353 & Sporobolus diander (Retz.) P. Beauv. & Bina joni & Poaceae & $\mathrm{M}$ & $\mathrm{H}$ & Anwar-0049 \\
\hline 354 & Sporobolus indicus (L.) R Br. & Lamba joni & Poaceae & M & $\mathrm{H}$ & Anwar-0412 \\
\hline 355 & Stemona tuberosa Lour. & $\begin{array}{l}\text { Lalgurania } \\
\text { alu }\end{array}$ & Stemoniaceae & M & $\mathrm{H}$ & Anwar-0205 \\
\hline 356 & Stenochlaena palustris (Burm. f.) Bedd & Dhekia lata & Blechnaceae & $\mathrm{F}$ & $\mathrm{H}$ & Anwar-1016 \\
\hline 357 & Stephania glabra (Roxb.) Miers & $\begin{array}{l}\text { Thanda } \\
\text { manik }\end{array}$ & $\begin{array}{l}\text { Menispermacea } \\
\mathrm{e}\end{array}$ & $\mathrm{D}$ & $\mathrm{CH}$ & Anwar-0733 \\
\hline 358 & Sterculia villosa Roxb. ex Smith & Udal & Sterculiaceae & $\mathrm{D}$ & $\mathrm{T}$ & Anwar-0890 \\
\hline 359 & Stereospermum suaveolens (Roxb.) DC & Muskanda & Bignoniaceae & $\mathrm{D}$ & $\mathrm{T}$ & Anwar-0934 \\
\hline 360 & Streblus asper Lour. & Shewra & Moraceae & $\mathrm{D}$ & $\mathrm{T}$ & Anwar-0683 \\
\hline 361 & Strobilanthes scaber Nees & $\begin{array}{l}\text { Kashkasatoit } \\
\text { a }\end{array}$ & Acanthaceae & $\mathrm{D}$ & $\mathrm{H}$ & Anwar-0680 \\
\hline 362 & Suregada multiflora (A. Juss.) Baill & Ban latkon & Euphorbiaceae & $\mathrm{D}$ & $\mathrm{T}$ & Anwar-0450 \\
\hline 363 & Swintonia floribunda Griff. & Boilam & Dipterocarpaceae & $\mathrm{D}$ & $\mathrm{T}$ & Anwar-1028 \\
\hline 364 & Synedrella nodiflora (L.) Gaertn & Nakphul & Asteraceae & $\mathrm{D}$ & $\mathrm{H}$ & Anwar-0820 \\
\hline 365 & Syzygium cumini (L.) Skeels & Kalojam & Myrtaceae & $\mathrm{D}$ & $\mathrm{T}$ & Anwar-1007 \\
\hline 366 & Syzygium balsameum (Wight) Walp & Khudijam & Myrtaceae & $\mathrm{D}$ & ST & Anwar-0761 \\
\hline 367 & Syzygium cerasoides (Roxb.) Raizada & Boti jam & Myrtaceae & $\mathrm{D}$ & ST & Anwar-0939 \\
\hline 368 & Syzygium formosum (Wall.) Masamune & Paniya jam & Myrtac & $\mathrm{D}$ & ST & Anwar-0980 \\
\hline 369 & Syzygium fruticosum DC. & Khudijam & Myrtaceae & $\mathrm{D}$ & $\mathrm{T}$ & Anwar-0051 \\
\hline 370 & Tabernaemonta & $\therefore$ & Apocyn & $\mathrm{D}$ & $\mathrm{S}$ & ar-0017 \\
\hline 371 & Tabernaemontana divaricata (L). R. Br & Tagar & naceae & $\mathrm{D}$ & S & Anwar-0011 \\
\hline 372 & Tacca integrifolia Ker-Gawl. & $\mathrm{Ba}$ & Tace & M & $\mathrm{H}$ & $r-0617$ \\
\hline 373 & Tamarindus indica $\mathrm{L}$. & Tatul & $\mathrm{Ca}$ & $\mathrm{D}$ & $\mathrm{T}$ & $r-1038$ \\
\hline 374 & $\begin{array}{l}\text { Tamilnadia uliginosa (Retz.) Tirveng. \& } \\
\text { Sastre }\end{array}$ & Pedalu & Rubiaceae & $\mathrm{D}$ & $\mathrm{T}$ & Anwar-0967 \\
\hline 375 & Tectona grandis L. f. & Segun & & $\mathrm{D}$ & $\mathrm{T}$ & -0933 \\
\hline 376 & Tecoma stans (L.) Juss. ex Kunth & $\begin{array}{l}\text { dey } \\
\text { ma }\end{array}$ & Bignoniaceae & $\mathrm{D}$ & S & Anwar-0029 \\
\hline 377 & $\begin{array}{l}\text { Tectaria chattagramica (Clarke) Ching \& } \\
\text { Sinensia }\end{array}$ & $\begin{array}{l}\text { Jungli } \\
\text { dhekia }\end{array}$ & Tectariaceae & $\mathrm{F}$ & $\mathrm{FH}$ & Anwar-0026 \\
\hline 378 & Tephrosia alba Du Puy \& Labat & Ban nil & Fabac & $\mathrm{D}$ & S & Anwar-1022 \\
\hline 379 & $\begin{array}{l}\text { Terminalia arjuna (Roxb. ex DC.) Wight } \\
\text { \& Arn. }\end{array}$ & Arjun & Combretaceae & $\mathrm{D}$ & $\mathrm{T}$ & Anwar-0975 \\
\hline 380 & Terminalia bellirica (Gaertn.) Roxb. & Dow & Combretaceae & $\mathrm{D}$ & $\mathrm{T}$ & Anwar-1020 \\
\hline 381 & Terminalia catappa $\mathrm{L}$. & Katbadam & Combretaceae & $\mathrm{D}$ & $\mathrm{T}$ & Anwar-0992 \\
\hline 382 & Terminalia chebula Retz. & Haritoki & Combretaceae & $\mathrm{D}$ & $\mathrm{T}$ & Anwar-0907 \\
\hline 383 & $\begin{array}{l}\text { Tetrastigma leucostaphyllum (Dennst.) } \\
\text { Alston ex Mabb }\end{array}$ & Horina lata & Vitaceae & $\mathrm{D}$ & $\mathrm{CH}$ & Anwar-0764 \\
\hline 384 & Themeda arundinacea (Roxb.) Ridl. & $\begin{array}{l}\text { Arunmeda } \\
\text { ghas }\end{array}$ & Poaceae & M & $\mathrm{H}$ & Anwar-1004 \\
\hline 385 & Themeda tremula (Steud.) Hack. & $\begin{array}{l}\text { Mulameda } \\
\text { ghas }\end{array}$ & Poaceae & M & $\mathrm{H}$ & Anwar-1024 \\
\hline 386 & $\begin{array}{l}\text { Themeda strigosa (Ham. ex Hook.f.) } \\
\text { A.Camus }\end{array}$ & $\begin{array}{l}\text { Gosameda } \\
\text { ghas }\end{array}$ & Poaceae & M & $\mathrm{H}$ & Anwar-0918 \\
\hline 387 & Thevetia peruviana (Pers.) K. Schum & Kolkephul & & $\mathrm{D}$ & ST & 014 \\
\hline 388 & Thuja orientalis L. & Thuja & Cupressaceae & $\mathrm{G}$ & $\mathrm{S}$ & Anwar-1003 \\
\hline 389 & $\begin{array}{l}\text { Thunbergia grandiflora (Roxb. ex Rottler) } \\
\text { Roxb. }\end{array}$ & Nilghonto & Acanthaceae & $\mathrm{D}$ & $\mathrm{C}$ & Anwar-0773 \\
\hline 390 & Thysanolaena maxima (Roxb.) O. Kuntze & Jharu ful & Poaceae & M & $\mathrm{H}$ & Anwar-0759 \\
\hline
\end{tabular}




\begin{tabular}{|c|c|c|c|c|c|c|}
\hline $\begin{array}{l}39 \\
1\end{array}$ & Tinospora crispa (L.) Hook. f. \& Thorns & Gulancha & Menispermiaceae & $\mathrm{D}$ & $\mathrm{CH}$ & Anwar-1025 \\
\hline $\begin{array}{l}39 \\
2\end{array}$ & Tinospora sinensis (Lour.) Merr & $\begin{array}{l}\text { Padma } \\
\text { gulancha }\end{array}$ & Menispermiacee & $\mathrm{D}$ & $\mathrm{CH}$ & Anwar-0943 \\
\hline 393 & Tournefortia viridiflora C. B. Clarke & Shamshog & Boraginaceae & $\mathrm{D}$ & $\mathrm{H}$ & Anwar-0585 \\
\hline 394 & Tragia involucrata $\mathrm{L}$. & Bichuti & Euphorbiaceae & $\mathrm{D}$ & $\mathrm{S}$ & Anwar-1015 \\
\hline 395 & Trema orientalis (L.) Blume & Banjiga & Ulmaceae & $\mathrm{D}$ & $\mathrm{T}$ & Anwar-0944 \\
\hline 396 & Trema tomentosa (Roxb.) Hara & Jinal & Ulmaceae & $\mathrm{D}$ & $\mathrm{T}$ & Anwar-1013 \\
\hline 397 & Tridax procumbens $\mathrm{L}$. & Tridhara & Asteraceae & $\mathrm{D}$ & $\mathrm{H}$ & Anwar-0301 \\
\hline 398 & Triumfetta rhomboidea Jacq. & Ban okra & Tiliacea & $\mathrm{D}$ & $\mathrm{H}$ & Anwar-0576 \\
\hline 399 & Urena lobata $\mathrm{L}$. & Banghagra & Malvaceae & $\mathrm{D}$ & $S$ & Anwar-0740 \\
\hline 400 & Vernonia cinerea (L.) Less. & Shial muti & Asteraceae & $\mathrm{D}$ & $\mathrm{H}$ & Anwar-0810 \\
\hline 401 & Vetiveria zizanioides (L.) Nash & Bena & Poaceae & M & $\mathrm{H}$ & Anwar-0948 \\
\hline 402 & Vigna pilosa (Willd.) Baker & Jikhrai & Fabaceae & $\mathrm{D}$ & $\mathrm{CH}$ & Anwar-0541 \\
\hline 403 & Vitex peduncularis Wall. ex Schauer & Harina & Verbenaceae & $\mathrm{D}$ & $\mathrm{T}$ & Anwar-0117 \\
\hline 404 & Vitex negundo L. & Nishinda & Verbenaceae & $\mathrm{D}$ & ST & Anwar- 0136 \\
\hline 405 & Woodfordia fruticosa (L.) Kurz & Dhatriphul & Rubiaceae & $\mathrm{D}$ & S & Anwar-0605 \\
\hline 406 & Wrightia arborea (Dennst.) Mabb. & Ruigach & Apocynaceae & $\mathrm{D}$ & ST & Anwar-0719 \\
\hline 407 & Xylia dolabriformis Benth & Loha kat & Fabaceae & $\mathrm{D}$ & $\mathrm{T}$ & Anwar-0955 \\
\hline 408 & Zanthoxylum rhetsa (Roxb.) DC. & Bajna & Rutaceae & $\mathrm{D}$ & $\mathrm{T}$ & Anwar-1034 \\
\hline 409 & Zizyphus glabrata Heyne ex Roth & Jangli-kul & Rhamnaceae & $\mathrm{D}$ & $\mathrm{T}$ & Anwar-0971 \\
\hline 410 & Zizyphus mauritiana Lamk & Boroi & Rhamnaceae & $\mathrm{D}$ & $\mathrm{T}$ & Anwar-1037 \\
\hline 411 & Zizyphus xylopyrus (Retz.) Willd. & Jangli boroi & Rhamnaceae & $\mathrm{D}$ & $S$ & Anwar-0968 \\
\hline 412 & Zizyphus oenoplia (L.) Mill. & Ban boroi & Rhamnaceae & $\mathrm{D}$ & $\mathrm{S}$ & Anwar-0977 \\
\hline
\end{tabular}

Legend: $\mathrm{C}=$ Climber, $\mathrm{CH}=$ Climbing Herb, $\mathrm{CS}=$ Climbing Shrub, $\mathrm{D}=\mathrm{Dicot}, \mathrm{F}=\mathrm{Fern}, \mathrm{FH}=\mathrm{Fern}$ Herb, $\mathrm{G}=$ Gymnosperm, $\mathrm{M}=$ Monocot, $\mathrm{SS}=$ Scandens Shrub, $\mathrm{ST}=$ Small Tree, $\mathrm{T}=$ Tree.

Acknowledgement: The first athor is gateful to the University Grants Commission (UGC) for granting Ph.D. research grant. Thanks are due to Mr. Abdur Rahim, Department of Botany, Jahangirnagar University and to Dr. Sarder Nasir Uddin, Senior Scientific Officer, Bangladesh National Herbarium for their help to identify the plant spcies.

\section{REFERENCES}

Ahmed, Z.U., Begum, Z.N.T., Hassan, M.A. Khondker, M.M., Kabir, S.M.H., Ahmad, M., Ahmed, A.T.A., Rahman, A .K.A. and Haque, E.U. (eds.). 2008 -2009. Encyclopedia of Flora and Fauna of Bangladesh. 6-10. Angiosperms; Dicotyledons. Asiatic Society of Bangladesh, Dhaka.

Alam, M.K. 2001 Development Plan for Sitakunda Botanical Garden and Eco-park. Office of the Project Director and Conservator of Forests, Chittagong Circle, Forest Department, Nandan Kanon, Chittagong, Bangladesh.

Alexiades M.N. 1996. Standard techniques for collecting and preparing herbarium species. A Field Manual. New York Botanical Garden. New York. pp. 99-126

Arefin, M.K., Rahman, M.M., Uddin, M.Z. and Hassan, M.A., 2011. Angiosperm flora of Satchari National park, Habiganj, Bangladesh. Bangladesh J. Plant Taxon. 18(2): 117-140.

Barua, S.P., Khan, M.M.H. and Reza, A.H.M.A. 2001. The status of alien invasive species in Bangladesh and their impact on the ecosystems. In: Balakrishna, P. (ed.), Alien invasive species. Report of workshop on alien invasive species. IUCN Regional Biodiversity Programme of Asia, Colombo, Sri Lanka.1-7 pp. 
BBS. 1997. Statistical Pocket Book of Bangladesh. Bangladesh Bureau of Statistics, Government of Bangladesh.

Braun-Blanquet, J. 1932. Plant sociology: The study of plant communities. McGeaw-Hill Book Co. Inc., New York, USA.

Cain, S.A. 1938. The species-area curve. American Midland Naturalist. 19: 573-581.

Chowdhury, Q.I. (ed.) 2001. The State of Sundarbans. 1st edition, Dhaka: Forum of Environmental Journalist of Bangladesh. pp. 5-16.

Hadi, M.A. and Rahman, M.M. 2013. Edaphic properties of the soils of Tilagarh Ecopark in Sylhet, Bangladesh. Physiol. Ecol. \& Environ. Sci. 4(1\&2): 37-43

Hooker, J.D. 1872-1897. The Flora of British India. 1-7. (Ind. Repr. 1973), Bishen Singh Mahendra Pal Singh, Dehra Dun, India.

Hossain, M.K. 2001. Overview of the forest biodiversity in Bangladesh. In: Assesment, conservation and sustainable use of forest biodiversity (CBD Technical series no. 3). SCBD, Montril, Canada. pp. 33-35.

Huq, S. and Asaduzzaman, M. 1999. Overview. In: Huq, S., Karim, Z., Asaduzzaman, M. and Mahtab, F. (eds.). Vulnerability and adaptation to climate change for Bangladesh. Kliwer Academic Publisher, Dordrecht, Netherlands. pp. 1-11.

Hyland, B.P.M. 1972. A technique for collecting botanical specimens in rain forest. Flora Malesiana Bulletin. 26: 2038-2040.

IPNI. 2008. The International Plant Names Index. <www.ipni.org>. Retrieved on 10 May 2015.

Islam, M.M., Amin, A.S.M.R. and Sarker, S.K. 2003. National Report on Alien Invasive Species of 'Bangladesh.' In: Pallewatta, N., Reaser, J.K. and Gutierrez, A.T. (eds.). Invasive Alien Species in South-Southeast Asia: National Reports \& Directory of Resources. Global Invasive Species Programme, Cape Town, South Africa. pp. 7-24.

Jain, S.K. and Raw, R.R. 1977. A handbook of field and herbarium methods/ xvi, 157 p.

Khan, M.S. 1977. Flora of Bangladesh, Report 4, Camelinaceae, Bangladesh National Herbarium, Bangladesh Agriculture Research Council (BARC), Farmgate, Dhaka.

Kent, M. and Coker, P. 1992. Vegetation description and analysis: A practical approach. The CRC Press, Inc. Belhaven Press London. pp 363.

Misbahuzzaman, K. and Alam, M.J. 2006. Ecological Restoration of Rainforest through Aided Natural Regeneration in the Denuded Hills of Sitakunda, Chittagong, Bangladesh. International Journal of Agriculture \& Biology. 8(6): 778-782.

Mittermeier, R.A., Myers, N., Thomsen, J.B., Da Fonseca, G.A. and Olivieri, S. 1998. Biodiversity hotspots and major tropical wilderness areas: approaches to setting conservation priorities. J. Conservation Biology. 12: 516-520.

Nishat, A., Huq, Imamul. S.M., Barua, S.P., Reza, A.A.H.M., Khan, M.A.S. (eds.). 2002. Bioecological zones of Bangladesh. IUCN, Bangladesh. pp.141

Prain, D. 1903. Bengal Plants. Vol. 1 and 2. Reprint Edition 1963. Calcutta. pp. 1-1319.

Raunkiaer, C. 1934. The Life-form of plants and statistical plant geography. Claresdon Press, Oxford.

Rashid, H.E. 1991. Geography of Bangladesh. University Press Ltd., Dhaka.

Shamsuddoha, M. and Nasir, T. 2011. Ecotourism: A Descriptive Study on Sitakunda Ecopark in Chittagong Division of Bangladesh. International Journal of Educational Research and Technology. 2(1): 08-13.

Shannon, C.F. and Wiener, W. 1963. The mathematical theory of communication. University of Illinois Press, Urbana.

Siddiqui, K.U., Islam, M.A., Ahmed, Z.U. Begum, Z.N.T., Hassan, M.A., Khondker, M.A.M. Rahman, M.M., Kabir, S.M.H., Ahmad, M., Ahmed, A.T.A., Rahman, A.K.A. and Haque, E.U. (eds.). 2007. Encyclopedia of Flora and Fauna of Bangladesh. 11. Angiosperms; Monocotyledons. Asiatic Society of Bangladesh, Dhaka. 
Simpson, E.H. 1949. Measurement of diversity. Nature 163 (30 April 1949): 688.

TROPICOS. 2010. Missouri Botanical Garden. <www.tropicos.org>. Missouri Botanical Garden, Saint Louis, Missouri, USA. Retrieved on 10 May 2015.

Uddin, M.Z. and Hassan, M.A. 2010. Angiosperm diversity of Lawachara National Park (Bangladesh): A preliminary assessment. Bangladesh J. Plant Taxon. 17 (1): 9-22.

Uddin, S.B. and Rahman, M.A. 1997. Angiospermic flora of Sitakunda in Chittagong, Bangladesh. Bangladesh J. Plant Taxon. 4(1): 17-36.

Wu, C.Y., Raven, P.H. and Hong, D.Y. (Eds) 1995-2013. Flora of China. Vols 2-25. Science Press and Missouri Botanical Garden Press, Beijing and St. Louis. 